\title{
How Well Do Response Changes of Striate Neurons Signal Differences in Orientation: A Study in the Discriminating Monkey
}

\author{
R. Vogels and G. A. Orban \\ Laboratorium voor Neuro- en Psychofysiologie, Katholieke Universiteit te Leuven, Herestraat, B 3000 Leuven, Belgium
}

Just-noticeable differences (JNDs) in orientation were measured in 2 monkeys using a temporal same-different task, with stationary rectangular gratings as stimuli, and compared to those of humans tested in the same setup. The JNDs of one monkey $\left(1.5^{\circ}\right)$ were similar to those of humans; those of the other monkey were larger $\left(5.8^{\circ}\right)$.

We recorded from $V 1$ neurons in these monkeys while they were performing the orientation discrimination with the same stimuli and under the same conditions as used in the behavioral testing. In order to determine how small a difference in orientation the $\mathrm{V} 1$ neurons can, in these conditions, signal reliably by small changes in firing rate, we performed 2 different receiver-operating characteristic (ROC) analyses. One ROC analysis was performed on the number of spikes evoked by the first of the 2 stimulus presentations as a function of the orientation of this stimulus. Neural JNDs derived from the neurometric curve were obtained in this way for $\mathbf{5 0}$ cells. In the second ROC analysis, the difference in the number of spikes evoked by the 2 stimuli presented in succession during 1 trial was anaiyzed as a function of orientation difference between the 2 stimuli. Neural JNDs were obtained by this procedure for 21 cells. These 2 complementary ROC analyses yielded very similar results. Also, the results were similar for the 2 monkeys. A minor fraction of $V 1$ cells can reliably signal difference in orientation as small as $2.5^{\circ}$, but none could signal differences smaller than $2^{\circ}$.

Our results also showed that the neural JND obtained by the ROC analysis depends on the duration of the interval during which spikes are counted. In these experiments, this duration could be chosen rather precisely, because the reaction times of the 2 monkeys were measured. Also, our results showed that the neural JND depends on the point of the tuning curve at which the measurement is made and is smallest when this is done on the steepest part of the tuning curve. Finally, our results show that the discriminative capacity of $V_{1}$ neurons does not depend so much on each of the tuning characteristics-bandwidth, response strength, and variability-as on the combination of these factors.

\footnotetext{
Received Dec. 19, 1989; revised June 5, 1990; accepted June 18, 1990.

This study was supported by grant GOA 84/88-62 of the Belgian Ministry of Sciences to G.A.O. R.V. was supported by the National Research Council of Belgium. We are much indebted to P. H. Schiller and N. K. Logothetis for providing the software and to $\mathrm{D}$. Tigwall for teaching the production of elgiloy electrodes. We thank G. Vanparrijs for drawing the figures; P. Kayenbergh and $G$. Meulemans for technical assistance; and $Y$. Celis for typing the manuscript. W. Spileers helped with the surgery and participated in some of the recording sessions. The critical reading of a draft of this paper by $S$. Raiguel, E. Vandenbussche, and $P$. De Weerd is gratefully acknowledged.

Correspondence should be addressed to Guy A. Orban, Laboratorium voor Neuro-en Psychofysiologie, Katholieke Universiteit te Leuven, Campus Gasthuisbcrg, Hercstraat, B 3000 Leuven, Belgium.

Copyright (C) 1990 Society for Neuroscience $0270-6474 / 90 / 113543-16 \$ 03.00 / 0$
}

Given the complexity of the mammalian, and especially the primate, brain, we need simplifying strategies when attempting to link neuronal properties and visual behavior. Orban et al. (1990) have suggested that the study of simple visual discriminations, in which subjects discriminate values of a single parameter (e.g., speed or orientation of an elongated contour) represents such a strategy. Such discriminations are simple for 3 rcasons. They arc restrictcd in timc: rcaction times are smaller than $500 \mathrm{msec}$, and only activity in the brain within this window has to be considered. They are also limited spatially, that is, only a restricted number of visual structures are involved. For orientation discrimination in the cat, we were able to show that areas 17 and 18 were required (Orban et al., 1990), but not the cortical areas receiving input from areas 17 and 18 (Vandenbussche el al., 1989). Finally, with adequate controls it is possible to ensure that subjects use only 1 cue-in the case of orientation discrimination, the orientation of the contour. Hence, the discrimination performance can be related to the activity of orientation-selective cells.

A number of recent studies conducted in anesthetized and paralyzed animals have suggested that the 'best cells' in the striate cortex can signal differences in a visual parameter as small as can be discriminated behaviorally (Parker and Hawken, 1985; Swindale and Cynader, 1986; Bradley et al., 1987; Skottun et al., 1987). In particular, Bradley et al. (1987) showed that cat striate cells, though broadly tuned for orientation, can signal changes in orientation as small as $2^{\circ}$ by virtue of small but reliable differences in strength of response to these stimuli. These authors concluded that the just-noticeable differences (JNDs) in orientation of the best cells were in close agreement with the JNDs measured behaviorally in cats.

We reexamined whether visual cortical cells are able to signal differences in orientation as small as JNDs in orientation measured behaviorally, because a number of questions can be raised as to the validity of the above-referenced physiological studies. First, all these studies have been conducted on cats or monkeys that were paralyzed and anesthetized during the single-cell recording. In order to measure the discriminative capacity of a single neuron, in conditions similar to those used in behavioral experiments, it is necessary to record from the neuron while the animal is performing a discrimination.

A second problem with previous work is that, in the latter studies, analyses were performed on numbers of spikes counted within a relatively large time interval. For instance, Bradley et al. (1987) analyzed spike counts in a time window of $500 \mathrm{msec}$. However, neither cats nor monkeys require that much information for making their decisions, because their reaction time in an orientation discrimination task is shorter than $500 \mathrm{msec}$ (De Weerd et al., 1990; see Results). Of course, we do not know when which sort of information is used, but the intervals in 
which spikes are counted should be at least as short as the reaction time measured behaviorally.

A third problem with these studies is that the stimulus used in the physiological experiments differed from those used for the psychophysical measurements. For instance, Bradley et al. (1987) compared single-cell responses to drifting sine-wave gratings with JNDs in orientation measured behaviorally using lines as stimuli (Vandenbussche and Orban, 1983). A valid comparison of single-cell JNDs and behavioral JNDs requires that both are measured using the same stimuli.

A fourth problem is that Bradley et al. (1987) used moving stimuli. However, moving stimuli stimulate both direction-selective and orientation-selective mechanisms of single cells. This may result in inaccurate estimates of the orientation-tuning properties for stationary stimuli, which are used in the psychophysical studies. Furthermore, these investigators analyzed spike counts measured during the prolonged presentation (several cycles) of temporally modulated gratings. Steady-state responses to drifting gratings lack the transient response that may occur with flashed stationary gratings. The latter initial transient response component should be included in the analysis, because it obviously occurs also during the presentation of stationary stimuli in the psychophysical task.

A final problem is that Bradley et al. (1987) studied cat area 17 neurons even though ablation of area 17 has no influence on cat orientation discrimination, especially at medium or high contrast (Orban et al., 1988). Hence, one would need to know whether JNDs of cells in area 18, which operate in parallel with area 17 in this task (Orban et al., 1990), are also in quantitative agreement with behavioral thresholds.

These considerations led us to reinvestigate how well single striate cells can signal orientation differences using the monkey as an experimental model rather than the cat. First, monkeys are able to make finer orientation discriminations than cats, yet the orientation bandwidth of striate neurons is similar in the cat and monkey (De Valois ct al., 1982). Hence, the apparent discrepancy between tuning width and behavioral performance is even larger in the monkey than in the cat. Second, orientation discrimination in primates involves higher areas, such as inferotemporal cortex, because lesion of inferotemporal cortex impairs orientation discrimination (Dean, 1978). Thus, the cortical pathway involved in orientation discrimination seems to include more processing stages in the monkey than in the cat, in which cortical areas beyond areas 17 and 18 seem to be uninvolved (Vandenbussche et al., 1989). Hence, in the monkey, there is an elaboration of the sensorial representation used for the discrimination, which can be followed by recording in the different areas in the pathway leading up to inferotemporal cortex. Third, it has been shown that, in the macaque, activity in inferotemporal cortex depends on VI input (Rocha-Miranda et al., 1975). Therefore, it is very likely that striate orientationsclcctive cells are a necessary step in the elaboration of the sensorial representation used for orientation discrimination. This contrasts with the cat, in which area 17 is not required for fine discriminations (Orban et al., 1990). Finally, recording in the awake monkey is a well-established practice, and it is possible to record single cells while the monkey performs a discrimination (Newsome et al., 1989).

We therefore recorded from striate neurons and studied their orientation selectivity while the monkey was performing an orientation discrimination. As a reference, we measured behavioral JNDs using the same behavioral paradigm, stimulus, and ec- centricity in the visual field as that used for the physiological testing. The aim was indeed to assess the discriminative capacity of single cells in conditions matching as closely as possible those in which behavioral thresholds are measured. Behavioral reaction times were measured in the discrimination task and were used to set the time windows in which spikes were counted to measure the neuronal response. Neuronal responses are not always normally distributed, especially when they are small (Bradley et al., 1987; Vogels et al., 1989). Hence, responses were analyzed with the nonparametric receiver-operating characteristic (ROC) analysis (Cohn et al., 1975). This analysis was applied to 2 sets of responses measured in a same-different task. In this task, 2 gratings are presented in succession, and the monkey decides whether or not the 2 have the same orientation. In a first set of experiments, the orientation tuning was sampled in small steps with a fixed orientation difference, and responses to the first grating presentation were analyzed. In a second set, a range of orientation differences was presented at a single reference orientation, and the difference in response between the first and second presentation was analyzed. A preliminary account of these results has been previously presented (Vogels and Orban, 1989b).

\section{Materials and Methods}

Subjects. Two rhesus monkeys (Macaca mulatta) served as subjects. Monkey Ronnie was a juvenile male, while Loebas was an older, dominant male monkey. During the experiment, the monkeys were on a strict water-deprivation schedule but had dry food ad libitum. Monkey Ronnie still is in good condition and continues to be used in other experiments. Monkey Loebas, after participation in other experiments, was killed with an overdose of Nembutal and perfused with fixative. The recording site was anatomically verified as being $\mathrm{V} 1$

Training apparatus. The subjects were isolated in a dark, black-painted room (average luminance, $0.003 \mathrm{~cd} / \mathrm{m}^{2}$ ). In the adjoining room, the computer (PDP11/73), the eye-movement monitor, a microdrive, monitors, an oscilloscope, and a window discriminator were set up. The monkey was seated in a primate chair placed in the center of a pair of horizontally and vertically oriented coils. The monkey's head could be firmly fixed by means of a pin cemented to the skull. This headholder was held to the skull by means of titanium orthopedic screws. Eye movements were measured using the modified magnetic scleral-search coil technique (Judge et al., 1980). The monkey had a teflon-coated stainless-steel coil sutured onto the sclera, which was subcutaneously wired to a connector in the headholder. Implantation of the scleral coil and headholder was performed under ketamine and halothane anesthesia. The currents generating the magnetic fields, as well as the filtering and amplification of the signal, were provided by eye-movement monitor $2 \mathrm{i}$ BDl (Indiran Instruments Inc., Brookline, MA). The analog eyeposition signals were sampled at a rate of $200 \mathrm{~Hz}$ by the PDP11/73 microcomputer. The monkey's correct responses were immediately rewarded by drops of apple juice using a continuous reinforcement schedule. The amount of juice could be manipulated on-line by changing the duration of opening of the solenoid controlling the juice.

Stimuli. Stimuli were generated on a HP1332 cathode-ray tube (green phosphor) with a Picasso CRT Image Synthesizer (Innisfree Inc., Cambridge, MA). The orientation, contrast, phase, and position of the stimuli was controlled by the computer. For psychophysical testing, as well as for the single-cell studies, we used rectangular gratings as stimuli. Grating stimuli have several advantages. By phase shifting the grating during the discrimination trials, we avoided the possibility that the monkey used the position of the stripe ends as a cue for discrimination. Furthermore, receptive fields (RFs) are small in V1, and the large size of the grating (diameter, $8-10^{\circ}$ ) ensured that the cells would be stimulated despite small changes in fixation point, which are unavoidable even with control of eye movements. We used rectangular gratings because V1 cells show little dependence on the spatial frequency of rectangular gratings (Schiller et al., 1976), so that we would drive as many cells as possible with the standard stimulus.

The corners of the gratings were masked by means of a plastic plate with a circular hole. This mask ensured that the subject was unable to 
use nearby contours as cues for the discrimination. The diameter of the mask was $9 \mathrm{~cm}$, which corresponded to $9^{\circ}$ for the standard distance $(57$ $\mathrm{cm}$ ) between the monkey's eye and the display. By small changes in this distance, the mask diameter could be changed from $8^{\circ}$ to $10^{\circ}$. The spatial frequency of the grating was 2 cycles/degree, the Michelson contrast was $80 \%$, and the mean luminance was $7 \mathrm{~cd} / \mathrm{m}^{2}$. For the psychophysical experiments, the spatial phase of the grating was randomized over trials. During single-cell testing, the phase was fixed, except for a few tests in which we explicitly studied the effect of phase randomization. The grating was stationary for the psychophysical as well as the physiological experiments, except for a few cells that were studied with both stationary and drifting gratings.

The mask surrounding the stimulus also contained a number of computer-controlled red light-emitting diodes (LEDs), each of which could serve as a fixation point. We used small $\left(1-2^{\circ}\right)$ flickering spots to map the position of the RFs of the cells or of the multicellular background activity. The RF of the cell was approximately centered on the grating by selecting the appropriate LED as fixation point.

Discrimination paradigm. We used saccadic eye movements in the direction of the stimulus as the operant. This facilitates training of the animals, because no association between a novel response (e.g., bar pressing) and a stimulus has to be taught. Also, because saccadic eye movements have the shortest latencies of all motor responses, their latency provides the most accurate estimation of how long this stimulus is processed before the response takes place. The discrimination paradigm was a temporal same-different task. The onset of the fixation point marked the start of a trial. The monkey had to saccade to the fixation point within $3000 \mathrm{msec}$ after its onset; otherwise, the fixation point went off, and the intertrial interval $(2000 \mathrm{msec})$ started again. After a 1000 -msec fixation, a square-wave grating $\left(S_{1}\right)$ was presented parafoveally for $350 \mathrm{msec}$ (Fig. 1). Then, after an interstimulus interval of 300 msec, the grating was presented again at the same position $\left(\mathrm{S}_{2}\right)$. If the 2 gratings presented in succession differed in orientation (different-trial), the monkey had to make a saccade towards the grating within $500 \mathrm{msec}$ after its presentation. When both gratings had the same orientation (same-trial), the monkey was required to continue fixating the LED for $500 \mathrm{msec}$ after the presentation of the second grating. Because monkeys have quite short latencies in this sort of task (see Results), the $500-\mathrm{msec}$ 'decision time' is adequate.

Fixation inaccuracies and the saccades directed toward the stimulus were detected by the computer using windows centered on the fixation spot and stimulus, respectively. The dimensions of the fixation window were $2.5 \times 2.5^{\circ}$. We required the monkey, before responding, to maintain fixation for a short interval after $S_{2}$ was presented. Because we wanted to measure the saccadic latency, this interval had to be as short as possible, yet long enough to force the monkey to look at $S_{2}$. Therefore, we started with a short interval of $50 \mathrm{msec}$ during the training and observed the monkey's performance. For monkey Loebas, a longer interval was not necessary, because this monkey reached $90 \%$ correct with this setting, which is impossible without looking at $\mathbf{S}_{2}$. For monkey Ronnie, who was much more active, the interval had to be prolonged to $180 \mathrm{msec}$, which is just shorter than his saccadic latencies (see Results).

Training procedures. The monkeys were well trained in the orientation discrimination task. We will only give a brief outline of the training procedure. We used standard operant techniques and positive reinforcement only.

First, the monkey was taught to fixate an LED, then was reinforced for making saccadic eye movements to the onset of the parafoveally presented grating. Next, the monkey was required to saccade only after the orientation of the grating had changed. Then, trials in which the orientation of the grating remained the same and in which the monkey had to keep fixating were introduced. When the monkey had mastered this step, the interstimulus interval was gradually increased to $300 \mathrm{msec}$. At this point, the monkey had acquired the basic task; this required about $14 \mathrm{~d}$ of daily training. We gradually decreased the orientation difference between 2 successively presented gratings. When the monkey performed well for a $10^{\circ}$ difference, we trained further with a staircase procedure, in which the orientation difference is adapted to the monkey's performance, until threshold was reached. This was done for several reference orientations. There was little transfer between reference orientations.

Psychophysical testing. Before the single-cell recordings, we measured JNDs in orientation with a staircase procedure. We used a staircase procedure of Wetherill and Levitt (1965) that converged on an orien-

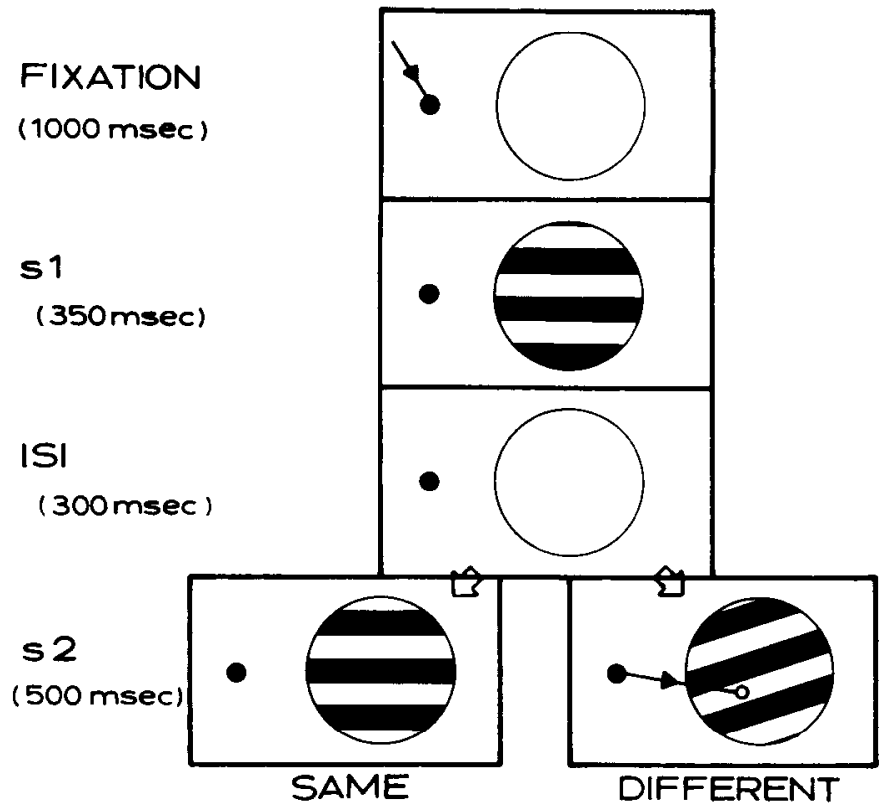

Figure 1. Description of orientation-discrimination task. After a $f x$ ation period of $1000 \mathrm{msec}$, the first stimulus $\left(S_{l}\right)$ is presented for 350 msec. After an interstimulus interval (ISI) of $300 \mathrm{msec}$, the second stimulus $\left(S_{2}\right)$ is presented at the same position as the first stimulus. If the stimuli differ in orientation (lower right), the monkey has to make a saccade to the grating within $500 \mathrm{msec}$. If the orientations arc the same (lower left), the monkey has to maintain fixation for $500 \mathrm{msec}$. When the monkey stops fixating the fixation spot or $500 \mathrm{msec}$ after $\mathrm{S}_{2}$ onset, whichever comes first, the stimulus and the fixation spot disappear, ending the trial. The stimulus was a stationary rectangular grating, centered $4-6^{\circ}$ from the fixation point.

tation difference corresponding to a $79 \%$-correct criterion. The orientation difference was decreased by $20 \%$ when the subject had correctly responded in 3 successive trials; the orientation difference was increased after each incorrect response. The geometric mean of the last 10 reversal points defined the JND in orientation. We used similar procedures to measure JNDs in orientation for the same stimuli in 3 well-trained human subjects. The only differences between the monkey threshold determinations and the human ones were that the latter subjects' eye positions were not monitored, and that they used a response key to indicate whether or not the sequentially presented gratings differed in orientation.

Single-cell recording. After the training and behavioral testing of the animals, a 19-mm-diameter stainless-steel chamber was implanted over the occipital lobe, partly covering the occipital ridge. Inside the chamber, a hole, about $1 \mathrm{~cm}$ in diameter, was drilled through the skull, leaving the dura mater intact. The craniotomy and implantation of the recording chamber was performed under ketamine and halothane anesthesia. During the single-cell recording sessions, a circular Plexiglas chamber was mounted on the steel well, and an electrode holder was fixed on top of this chamber. By rotating the Plexiglas chamber and/or the electrode holder, it was possible to reach any desired position in the chamber. The chamber was filled with sterile mineral oil and was sealed by the base of the electrode holder. The electrode was advanced more or less perpendicular to the brain using a hydraulic Kopf Micropositioner (Model 640, David Kopf Inc., Tujunga, CA). The signal from the electrode was amplified (WPI Instruments Inc.) and band-pass filtered (Wavetek Rockland Inc.). Spikes were isolated using a 2-level window discriminator (Indiran Instruments Inc.). The triggered spike was fed into the microcomputer (sampling rate, $1000 \mathrm{~Hz}$ ). We could monitor on-line the eye position with respect to the fixation point and stimulus position, stimulus-triggered spike trains, and the stimulus that was actually presented to the monkey. The PDP $11 / 73$ controlled the stimulus presentation and juice delivery, collected the spikes and eye movements, and displayed the eye position and the spike trains.

Single-cell recordings were performed daily for about 5 weeks in each animal. For each daily recording session, a fresh penetration was made 
with glass-coated elgiloy microelectrodes (Suzuki and Azuma, 1976) having an impedance ranging from 6 to $12 \mathrm{M} \Omega$. The penetrations were made in area V1 of the left hemisphere. The recording sessions usually lasted between 3 and $4 \mathrm{hr}$, depending on the performance of the monkey. The position of the RFs for a given penetration was usually plotted using multicellular activity (see Poggio et al., 1977). This was done manually by moving a flickering spot while the monkey was fixating the LED. When the monkey stopped working, we demounted the electrode-holder apparatus, cleaned the chamber with sterile saline, put a few drops of dexamethason and chloromycetin on the dura mater, then closed the chamber with a plastic cap. The animal was then returned to its home cage.

Physiological testing strategies. The first decision to be made was whether or not to adapt the stimulus to the RF properties of the cells. Simple cells are phase sensitive, end-stopped cells are sensitive to the grating dimension, disparity-selective cclls arc sensitive to the depth of the stimulus, and wavelength-selective cells are sensitive to the color of the stimulus. We decided not to adapt these parameters to the properties of the cell under study and to adjust the stimulus position and orientation only. Cells in different areas may have different stimulus requirements, and comparison between areas is facilitated when the same standard stimulus is used for all areas. Furthermore, because the stimulus is identical for the psychophysics and the physiology, we were indeed measuring the contribution of the neurons to the psychophysical task (see Discussion for further elaboration). Hence, the only adaptation of the stimulus was to center the stimulus on the RF and to optimize the orientation of the grating for the cell under study. In 3 cells, the variability obtained without phase randomization was compared to that obtained with phase randomization and was found to be similar.

In order to adapt the orientation of $S_{1}$ to the optimal orientation of the cell under study, we had to change the behavioral task of the monkey. Rather than working at a few reference orientations with orientation differences of different magnitude, as for the psychophysical testing, we trained the monkey to discriminate at all orientations but at the expense of using a constant, relatively large orientation difference. Hence, monkeys learned to perform 2 versions of the discrimination: one in which the orientation of the first stimulus was always the same and the difference between $S_{1}$ and $S_{2}$ was manipulated, and one in which the orientation of the first stimulus randomly varied and the difference between $S_{1}$ and $S_{2}$ was constant. The latter test, the variable-orientation task, was typically used to explore the orientation tuning of the cortical cells, using for $S_{1} 6$ or 12 orientations differing by 30 or $15^{\circ}$. The former task, the fixed-orientation task, was typically used for the psychophysical testing. In both the fixed-and the variablc-oricntation tasks, there were, on average, as many same and different trials, to avoid the possibility of the monkey developing a behavioral response bias. In both kinds of tests, the order of presentation was randomized. In the variable-orientation task, the order of presentation of $S_{1}$ orientations and of sameand different-trials were randomized; in the fixed-orientation task, the order in which the differences (including 0 difference) occurred was randomized. The monkeys found the variable-orientation task very difficult, even after determination of JNDs at 3 reference orientations, and both monkeys had to be given considerable ( 2 months) training in order to perform well in this task.

In order to test how well the neuron could signal small differences in orientation under conditions similar to those in which behavioral JNDs were measured, 2 complementary approaches were followed. The first approach (Table 1) was to use the variable-orientation task, but with small steps (typically $3.5^{\circ}$ rather than 15 or $30^{\circ}$ ) and to analyze the difference in responses to $S_{1}$. We analyzed the response to $S_{1}$ because the number of presentations was larger than that of $S_{2}$. Indeed, half of the trials were different-trials. It should be stressed that, in the variable task, the monkey cannot perform the task above chance without processing of $S_{1}$ because the same orientation of $S_{2}$ can correspond to a same-trial or a different-trial, depending on the orientation of $S_{1}$. This first approach has the advantage that the performance of the cell can be studied simultaneously at different points along the tuning curve, in fact, at all orientations tested for that cell. It implies, however, that the response of the neurons to $S_{1}$ and $S_{2}$ are equal. This was indeed the case for the majority of V1 cells (see Results). The other approach uses a fixed-orientation task at a selected point on the tuning curve of the neuron under study. This entails a drawback in that performance of the cell is measured only at that point. However, it allows one to take into account differences in response to $S_{1}$ and $S_{2}$. Furthermore, it avoids the contribution of long-term variability (Bradley et al., 1987) to the performance of the cell. The latter approach required further training of the monkey because it had to perform the fixed task at many different reference orientations. Only monkey Ronnie, who had far better overall performances than monkey Loebas, was trained for this difficult exercise.

Analysis of neuronal responses. The orientation tuning properties were derived from the analysis of the responses to $S_{1}$ in the variable-orientation task run with coarse steps (Table 1). The response strength and the variance of this mean response were evaluated by measuring the number of spikes in the $0.5 \mathrm{sec}$ following the presentation of $S_{1}$ and expressing it in terms of firing rate (Vogels et al., 1989). The bandwidth (tuning width at half height) was derived from the tuning curve in which the net response was plotted as a function of orientation and linearly interpolated. The net response was obtained by counting the number of spikes during a $400-\mathrm{msec}$ interval after presentation of $S_{1}$ and subtracting the number of spikes occurring during $400 \mathrm{msec}$ before $S_{1}$ Finally, the latency was calculated with a variant of the CUISUM method (Ellaway, 1978). Spikes were counted with a bin width of $20 \mathrm{msec}$ starting $200 \mathrm{msec}$ before grating onset. The spike counts of the 10 bins preceding grating onset were averaged to obtain an estimate of the average number of spikes within a 20 -msec bin with no grating present, that is, 'spontaneous activity.' For each bin during the grating presentation, we then computed the difference between the number of spikes in that bin and the spontaneous-activity spike count. Then CUSUM values were calculated by summing these differences according to the following formula:

$$
\operatorname{CUSUM}(n)=\sum_{i=1}^{n}[\operatorname{Diff}(i)]
$$

where $\operatorname{Diff}(n)$ is the difference between the number of spikes in bin $n$ and the spontaneous-activity bin. The variable $i$ is 1 for the first 20msec bin after the grating onset, 2 for the next $20-\mathrm{msec}$ bin, and so on. The start of a response corresponds to a sudden increase in the difference between subsequent CUSUM values. We defined the latency of the cell as the time period corresponding to the first bin $i$ for which (1) CU. SUM $(i)$ was larger than CUSUM $(i-1)$, (2) for which CUSUM $(i)$ was smaller than CUSUM $(i+1)$ and $\operatorname{CUSUM}(i+1)$ was smaller than CUSUM $(i+2)$, and (3) the difference between successive CUSUMs, that is, CUSUM $(i)$ - CUSUM $(i-1)$, was larger than the differences between CUSUMs of earlier bins. These rules were determined empirically and could be applied to all but a few dubious cases.

To analyze the cells' discriminative performance and to derive neuronal JNDs, we used the nonparametric receiver-operating characteristic (ROC) analysis in the manner of Cohn et al. (1975), Tolhurst et al. (1983), Bradley et al. (1987), and Newsome et al. (1989). For the variable-orientation task, we analyzed how the responses to $S_{1}$ changed with orientation. For the fixed-orientation task, we analyzed how the difference in response between $S_{2}$ and $S_{1}$ changed with orientation difference. The details of these analyses are given in the Results.

\section{Results}

This section is organized into 7 parts. First, we will compare the behavioral JNDs measured at 3 reference orientations in

Table 1. Physiological testing strategies

\begin{tabular}{lll} 
Task & Analysis & Result \\
\hline Variable-orientation task, coarse steps & Response to $S_{1}$ & Orientation tuning \\
Variable-orientation task, fine steps & Response to $S_{t}$ & Neural JND \\
Fixed-orientation task & Difference between responses to $S_{2}$ and $S_{1}$ & Neural JND
\end{tabular}


the 2 monkeys to those measured in humans. Second, we will report the behavioral performance of the 2 monkeys during physiological testing. The third part will compare the 3 parameters describing the orientation selectivity - bandwidth, response strength, and normalized variance - in those cells tested for their discriminative performance and in those cells representing a broader population of $183 \mathrm{~V} 1$ cells. The fourth part will describe the results of the ROC analysis of the $S_{1}$ responses obtained in the variable-orientation task. The fifth part will be devoted to the ROC analysis of the response difference between $S_{1}$ and $S_{2}$ obtained in the fixed-orientation task. The sixth part will compare the outcome of these 2 analyses in view of the response similarity to $S_{1}$ and $S_{2}$ for identical orientations and the contribution of long-term variability to the neuronal performance. In the final part, the ncural JNDs will be related to the 3 characteristics describing the orientation tuning.

\section{Behavioral thresholds}

Figure 2 shows the JNDs in orientation, determined with the staircase procedure and plotted as a function of reference orientation for the 2 monkeys and for 3 human subjects. The JNDs of monkey Ronnie are averages of at least 10 measurements, while those of monkey Loebas are averages of at least 6 measurements. These data were obtained after considerable training, 12 months per reference orientation. The human JNDs are averages of 3 subjects. Little training was required for human subjects. All JNDs were measured at an eccentricity of $6^{\circ}$, which is close to the average eccentricity of the single cells recorded. Human JNDs were measured only at the horizontal reference orientation and one oblique reference because previous work (Orban et al., 1984; Vogels and Orban, 1985) has shown that JNDs in orientation are similar at both principal reference orientations (horizontal and vertical) and at both oblique references. Human JNDs were very similar for all 3 subjects and slightly smaller at the horizontal $\left(0.95^{\circ}\right)$ than at the oblique $\left(1.8^{\circ}\right)$ reference. It is noteworthy that the human JNDs are not very different from those reported for central vision by Vandenbussche et al. (1986). This is not surprising because, in the latter study, JNDs in orientation, measured with stationary bars, changed little for eccentricity changes restricted to the central $10^{\circ}$ of the visual field. The JNDs of monkey Ronnie were of the same magnitude as the human JNDs $\left(1.45^{\circ}\right)$, but were equal at all 3 reference orientations. For this monkey, we tested JNDs in orientation over a range of grating diameters and found that JNDs were unaffected by a reduction to diameters as small as $2^{\circ}$. Those of monkey Loebas were larger, ranging between 5.1 and $6.2^{\circ}$, values close to the best monkeys in Dean's (1978) study. Although the standard procedure was to measure the JNDs with randomization of the stimulus phase, the same values were obtained when stimulus phase was constant.

\section{Behavioral performance during physiological testing}

To determine the behavioral JNDs, a staircase procedure was used in which the orientation difference was adapted to the monkey's performance. In contrast, the testing procedure used during physiological recording was nonadaptive. This, and the fact that the reference orientation changed during a single daily session, explains that the performance of the monkeys was not as acute as during behavioral JND determination. An important benefit of these nonadaptive procedures is that saccadic latencies can be determined, because only a restricted number of orientations are presented. Figure 3 shows a representative example

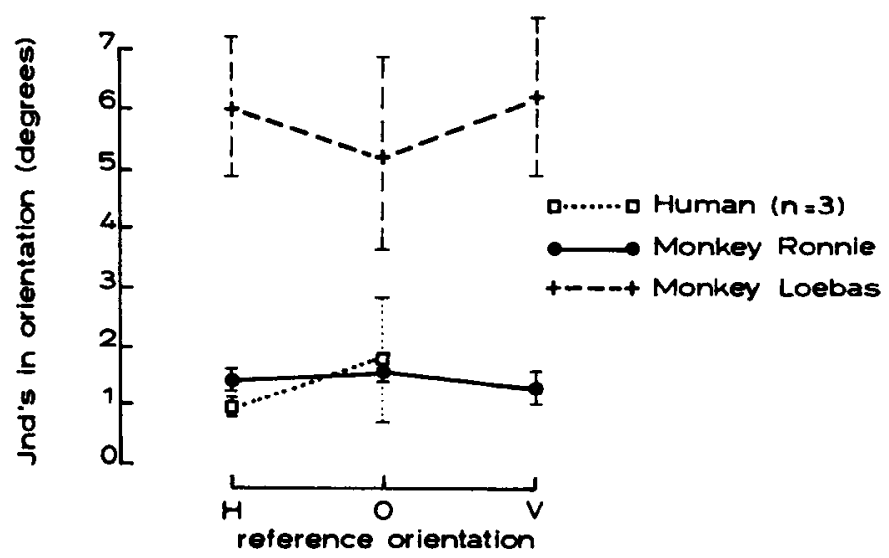

Figure 2. Just-noticeable difference (JND) in orientation as function of reference orientation compared in humans and monkeys. The thresholds $(79 \%$ correct) were measured with a staircase method. The human data are averages of 3 subjects. The monkey data are the average of at least 10 or 6 determinations in monkey Ronnie and monkey Loebas, respectively. The error bars represent the SDs of the average across subjects for human data and the SD per subject for the monkey data. Since this study has been completed, we have trained a third monkey for which the JNDs are similar to those of monkey Ronnie. They average about $2^{\circ}$ at each reference orientation.

of the psychometric curve obtained with the fixed-orientation task in both monkeys. In the psychometric curve, the percent of correct responses is plotted as a function of orientation difference, and the JND can be derived from the intersection of this curve with the 75\%-correct level. The saccadic latencies are surprisingly short, ranging near 200 msec for monkcy Ronnic and near $250 \mathrm{msec}$ for monkey Loebas. In both monkeys, the latencies tend to increase as the orientation difference decreases.

The monkeys' performance in the variable-orientation task cannot be summarized by a psychometric curve, because only 1 orientation difference was used. The performance is summarized instead by the proportion of correct responses for the orientation difference used ( $15^{\circ}$ for Ronnie and $90^{\circ}$ for Loebas). These measures did not depend on orientation and averaged $91 \%(\mathrm{SD}, 4 \%)$ for Ronnie and $81 \%(\mathrm{SD}, 12 \%)$ for Loebas. The saccadic latencies in this task were also independent of orientation and averaged $203 \mathrm{msec}(\mathrm{SD}, 13 \mathrm{msec}$ ) for Ronnie and $400 \mathrm{msec}(\mathrm{SD}, 43 \mathrm{msec}$ ) for Loebas.

\section{Response properties of cells tested for discriminative capacity}

Because the orientation tuning of monkey cells is wide compared to behavioral thresholds, we concentrated our testing on cells that might reasonably be expected to reliably signal small differences in orientation. In order to appreciate how great a role selection played in our samples, we compare the tuning characteristics of the cells of our sample to a larger sample of 183 cells tested with the variable-orientation task using coarse steps. The RFs of cells in both samples were located between $4^{\circ}$ and $6^{\circ}$ from the fovea, near the horizontal meridian. Although we have no precise information on laminar position, layer $4 \mathrm{C}$ can be recognized as a band of strong activity (Poggio et al., 1977), and almost all cells were recorded outside this layer.

Because the saccadic latencies are short, which implies a short processing period in the visual system, response latencies of cortical cells will also determine the discriminative capacity of cells. Indeed, a neuron with a short latency will fire more action potentials before the monkey responds than will a cell with a 

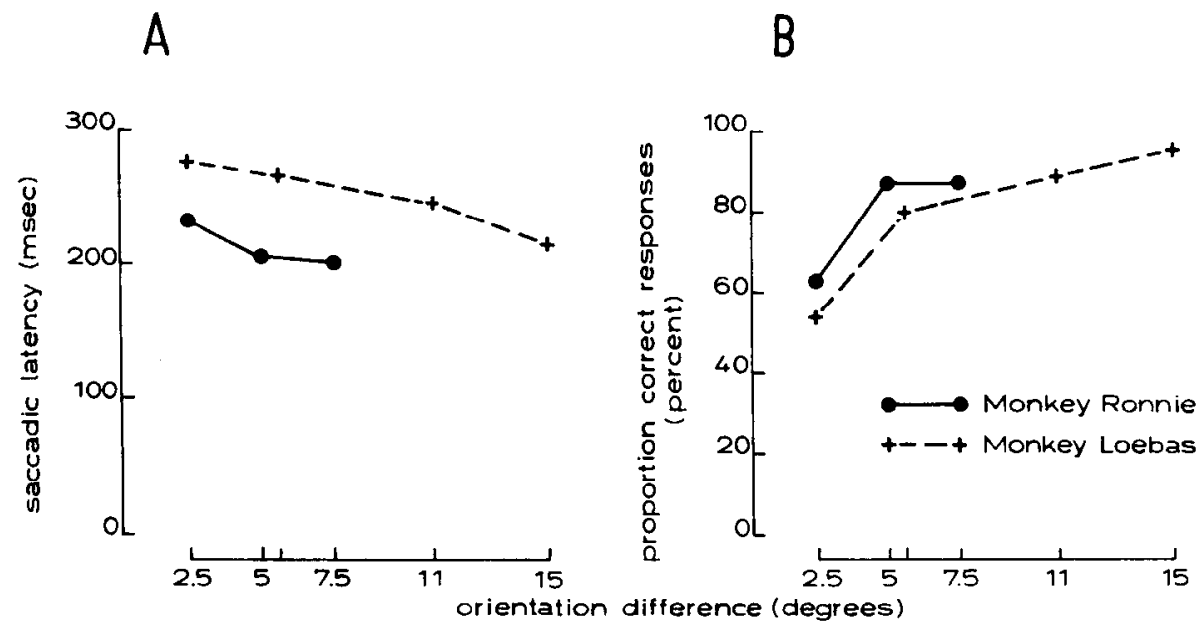

Figure 3. Saccadic latencies $(A)$ and proportion correct responses $(B)$ as function of orientation difference in fixed-orientation task. Representative data of monkeys Ronnie and Loebas obtained in a single determination are shown. Notice that the nonadaptive procedure yields slightly higher thresholds than the staircase procedure (compare Figure 2). long latency, and the discriminative capacity of cells increases with the number of action potentials (see below). Therefore, we will compare 4 characteristics that contribute to the performance of the neuron for the different samples. The first 2 characteristics are the orientation-tuning bandwidth and the response strength, both of which determine the slope of the tuning curve. Because the response variance is proportional to mean response strength (Vogels et al., 1989), the cells are characterized by the normalized variance obtained by dividing the variance by responsc strength. This characterization of variability, which is independent of the response strength, represents the third characteristic, and the neuronal latency, the fourth one.

The distributions of these characteristics for the larger sample are shown in Figure 4 . The bandwidth and response strength were very similar for both monkeys, the median bandwidth being $41^{\circ}$ and $39^{\circ}$ for Ronnie and Luebas, respectively, and the median response strength being 16 and 15 spikes/sec for Ronnie and Loebas, respectively (Table 2). Tested with the Mann-Whitney $U$ test, the normalized variance was significantly (MW $U=$ $3108, p<0.002$ ) smaller for Ronnie than for Loebas, the median values being 1.73 for Ronnie and 2.79 for Loebas. The latency was significantly shorter (MW $U=3707, p<0.00014$ ) for Ronnie than for Loebas, the median value being $60-80 \mathrm{msec}$ for Ronnie and 80-100 msec for Loebas (Table 2).

The distributions of these characteristics for the cells tested for their discriminative capacity are indicated by inset bars in Figure 4. Fifty cells (Ronnie, 31 cells; Loebas, 19 cells) were tested with the variable-orientation task using fine steps, and 21 cells were tested in Ronnie with the fixed-orientation task. On average, 15 trials were presented at each orientation in the variable-orientation task, and at least 10 trials at each nonzero orientation difference in the fixed-orientation task, yielding an average of 40 same- and different-trials for comparison. Orientation-tuning characteristics for the cells tested with the variable-orientation task using fine steps did not differ between monkcys (Table 2), and the difference in latency was barely significant (MW $U=208, p<0.04$ ). Compared to the larger sample, this sample was biased towards steeper tuning curves, because of smaller bandwidths in Ronnie and of stronger responses in Loebas, and to smaller variability and shorter latency, especially in Loebas. The sample of cells tested with the fixed-orientation task was more clearly biased than the sample of cells tested with the variable-orientation task using fine steps. Compared to the larger sample, the tuning curves of the cells tested in the fixedorientation task were steeper, their variability smaller, and latency shorter (Table 2).

\section{ROC analysis of differences in responses to $S$, signaling differences in orientation of $S_{\text {, }}$}

The ROC analysis is performed on the distributions of responses to different orientations. The number of spikes occurring in a fixed time interval after the onset of $S_{1}$ is taken as a mcasurc of response. This interval can be defined precisely in our experiments. Because no cell had a latency shorter than $25 \mathrm{msec}$, the

Table 2. Orientation-tuning characteristics and latencies of different samples

\begin{tabular}{|c|c|c|c|c|c|}
\hline & \multicolumn{2}{|c|}{ Variable orientation, coarse ${ }^{a}$} & \multicolumn{2}{|c|}{ Variable orientation, fine } & \multirow{2}{*}{$\begin{array}{l}\text { Fixed orientation, } \\
\text { Ronnie } \\
(n=21)\end{array}$} \\
\hline & $\begin{array}{l}\text { Ronnie } \\
(n=83)\end{array}$ & $\begin{array}{l}\text { Loebas } \\
(n=100)\end{array}$ & $\begin{array}{l}\text { Ronnie } \\
(n=31)\end{array}$ & $\begin{array}{l}\text { Loebas } \\
(n=19)\end{array}$ & \\
\hline \multirow[t]{2}{*}{ Bandwidth } & $41^{b}$ & 39 & 25 & 36 & 36 \\
\hline & $(18-74)$ & $(25-54)$ & $(11-44)$ & $(25-44)$ & $(19-51)$ \\
\hline \multirow[t]{2}{*}{ Response strength } & 16 & 15 & 14 & 24 & 51 \\
\hline & $(8-37)$ & $(8-32)$ & $(9-58)$ & $(9-39)$ & $(13-74)$ \\
\hline \multirow[t]{2}{*}{ Normalized variance } & 1.73 & 2.79 & 1.64 & 1.57 & 1.27 \\
\hline & $(1.14-3.72)$ & $(1.82-4.51)$ & $(1.09-3.64)$ & $(0.84-2.56)$ & $(0.89-2.79)$ \\
\hline \multirow[t]{2}{*}{ Latency } & $60-80$ & $80-100$ & $60-80$ & $60-80$ & $40-60$ \\
\hline & $(40-60$ to $80-100)$ & $(60-80$ to $100-120)$ & $(40-60$ to $60-80)$ & $(60-80$ to $80-100)$ & $(40-60$ to $60-80)$ \\
\hline
\end{tabular}

a The median bandwidth was calculated for 189 cells and the median latency for 206 cells rather than 183.

${ }^{b}$ Median and quartiles. 

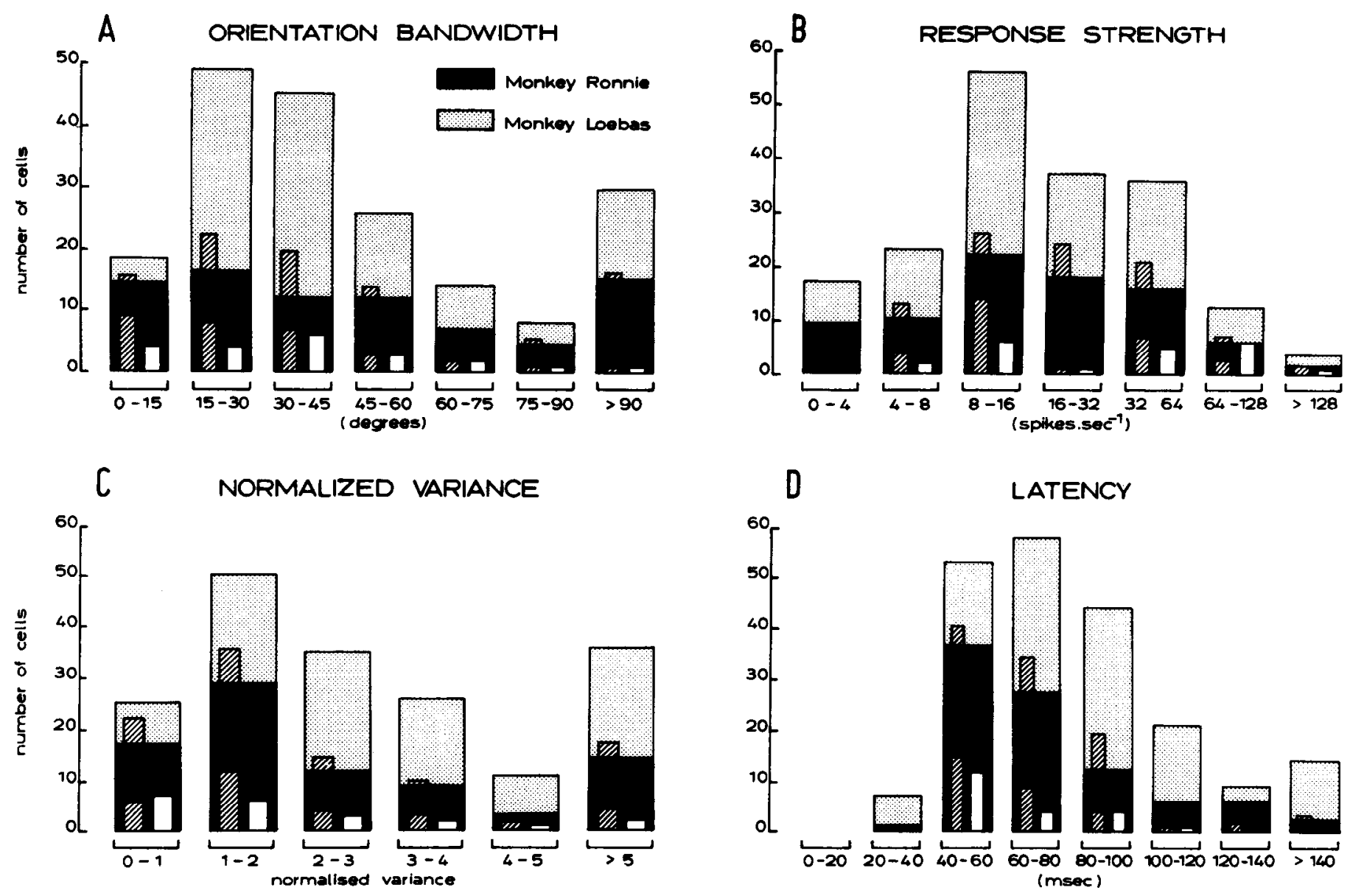

Figure 4. Distribution of orientation-tuning characteristics and of latency obtained with variable-orientation task using coarse steps. Distributions are shown for 3 samples: the larger sample $(n=183)$ measured in monkey Ronnie (black columns) and monkey Loebas (shaded columns); the sample tested with the variable-orientation task using fine steps $(n=50)$ shown as striped insets for each monkey separately, and the sample tested with the fixed-orientation task $(n=21)$ shown as open insets for monkey Ronnie.

interval starts $25 \mathrm{msec}$ after the onset of $\mathrm{S}_{1}$. We used the saccadic latency after collicular stimulation, which ranges between 20 and $30 \mathrm{msec}$ (Robinson, 1972), as a lower bound for the time needed by nervous signals to travel from V1 to the eye muscles. Because the shortest saccadic latencies of the monkeys were about $200 \mathrm{msec}$, the analysis interval ends at $175 \mathrm{msec}$ after $S_{1}$ onset to allow time for the signals to reach the eye muscles.

The ROC analysis determines the probability of correct responses for an ideal observer using only the firing of the cell under study to discriminate orientations. On average, this analysis is based on data from 30 trials. Such an analysis is illustrated in Figure 5 for a relatively narrowly tuned cell. The optimal orientation $\left(10.5^{\circ}\right)$, which elicits a larger average response than the orientations indicated by numbers 1,2 and 3 , is taken as reference, and the analysis determines the probability with which the reference can be distinguished from orientations 1,2 and 3 by using the rule that responses larger than the criterion indicate the reference orientation. The distributions of number of spikes elicited by the reference and orientation 1 are given in Figure $5 B$. These distributions are used to derive the proportion of hits and false alarms for a number of criteria ( 10 in the present cases). A hil is defined as a presentation for which the response exceeding the criterion belongs to the reference distribution, and a false alarm is defined as a presentation for which the response exceeding the criterion belongs to the distribution of orientation
1. These proportions are indicated for a large criterion $\left(\mathrm{C}_{7}\right)$ in Figure $5 B$ and a lower criterion $\left(C_{4}\right)$ in Figure $5 C$. For criterion $\mathrm{C}_{7}$, the proportion of hits and false alarms was $28 \%$ and $0 \%$, respectively. For criterion $\mathrm{C}_{4}$, which lies in the region of overlap of the 2 distributions, the proportion of false alarms was larger, $18 \%$, compared to $64 \%$ hits. Plotting the proportions of hits as a function of the proportion of false alarms for the 10 criteria yields the ROC curve of orientation 1, which is plotted in Figure $5 D$. Criteria outside the region of overlap of the distribution, such as $\mathrm{C}_{7}$, contribute points on the boundary of the diagram, while points corresponding to criteria in the region of overlap, such as $\mathrm{C}_{4}$, yield points inside the diagram. Comparison of the reference distribution with itself would yield $50 \%$ hits and $50 \%$ false alarms for all criteria. This corresponds to the positive diagonal marked $\mathrm{R}$ in the ROC diagrams. Complctcly nonoverlapping distributions yield an ROC curve that follows the left and upper boundary of the diagram. Hence, discrimination will be better when the ROC curve lies closer to the boundary of the diagram and further from the positive diagonal. Repeating the same analysis for the reference orientation and each of the other 2 orientations yields the ROC curves marked 2 and 3 in Figure $5 E$. Integrating the area under the ROC curve yields the proportion of correct responses of an ideal observer using the cell's responses (Green and Swets, 1966), which are plotted as a function of orientation in Figure $5 F$. This curve, in which 
Figure 5. ROC analysis of responses to $\mathrm{S}_{1} . A$, Orientation tuning curve of cell Loe 29 obtained with the variable-orientation task using fine steps. The median number of spikes measured in a 500-msec interval after $S_{1}$ onset and the 20 th and 80th percentiles are shown. The reference orientation $(R)$ and the 3 orientations that are compared to it $(1$, 2 , and 3 ) are indicated. $B$ and $C$. Distributions of numbers of spikes in a 150 msec interval starting $25 \mathrm{msec}$ after $\mathrm{S}_{1}$ onset for the reference orientation and orientation 1 compared to a large criterion $\left(C_{7}\right)$ in $B$ and a medium criterion $\left(C_{4}\right)$ in $C$. The 10 different criteria are indicated by vertical broken lines. The proportions of hits and false alarms for criteria $\mathrm{C}_{7}(B)$ and $\mathrm{C}_{4}(C)$ are indicated by shaded columns (to the right of the criterion) in distributions $R$ and 1 respectively. $D$, ROC curve obtained for the comparison of the reference orientation with orientation 1: each criterion corresponds to a point on the curve, but criterion 8 and 9 yield the same point because the bin in the distributions between these 2 criteria is empty. The points corresponding to criterion $\mathrm{C}_{7}(B)$ and criterion $\mathrm{C}_{4}(C)$ are indicated. Comparison of the reference with itself yields the positive diagonal labeled $R$ $E$, ROC curves obtained for the comparison of the reference orientation with itself and with orientations 1,2 and 3 . $F$, the neurometric curve plotting proportion correct as a function of the orientation difference with respect to the reference. The neural JND defined as the intersection of the neurometric curve with the $75 \%$-correct level is indicated.
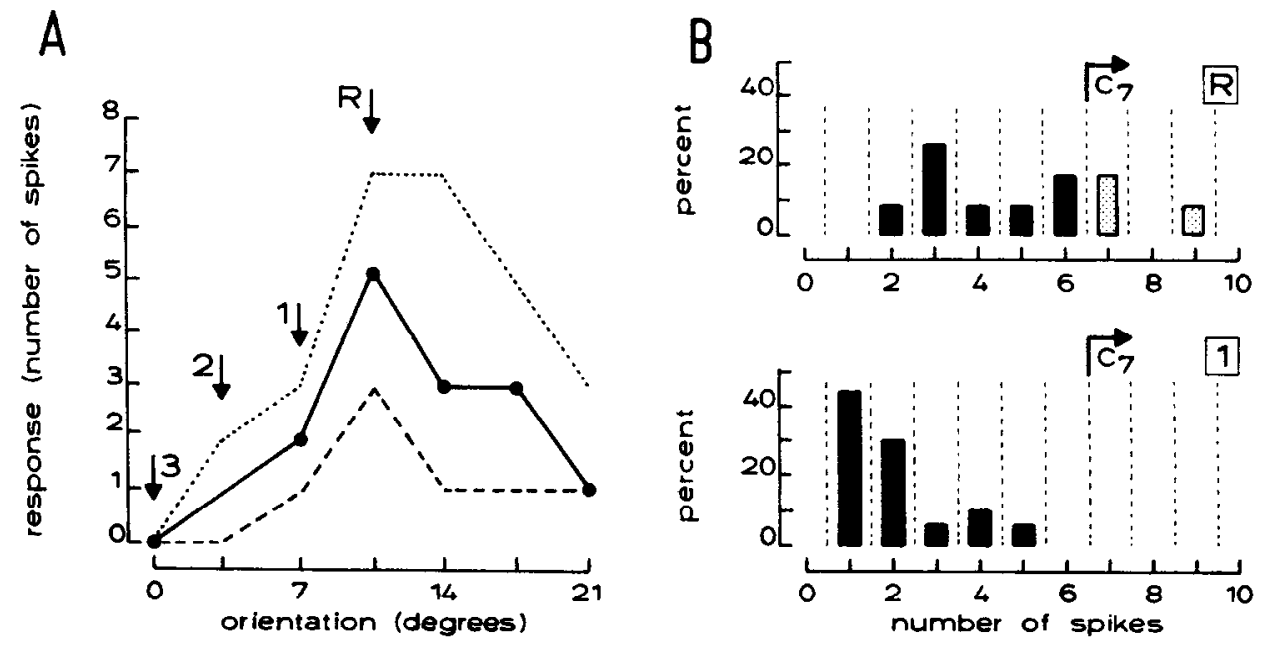

C
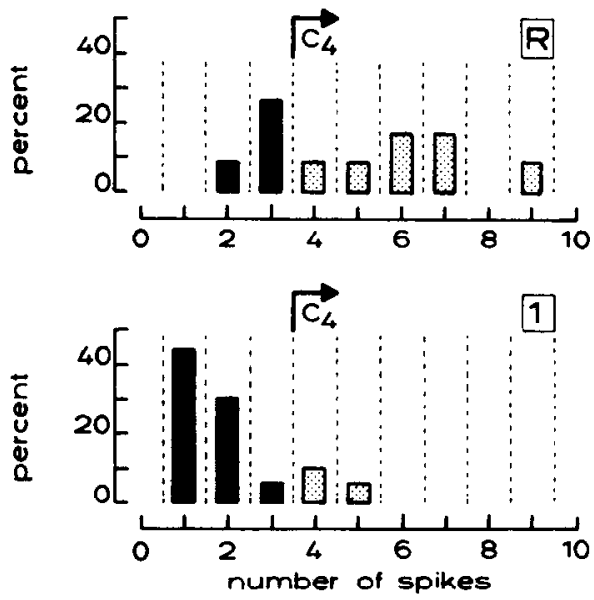

E

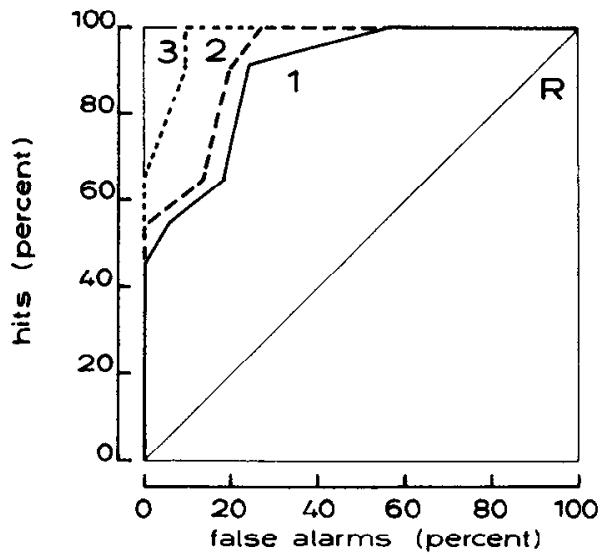

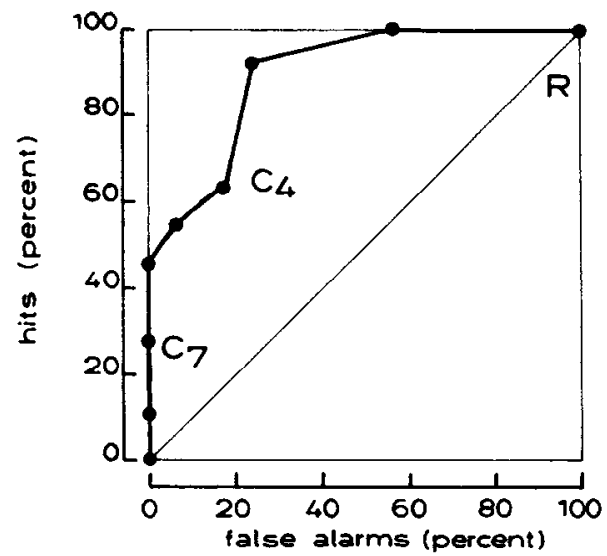

F

D

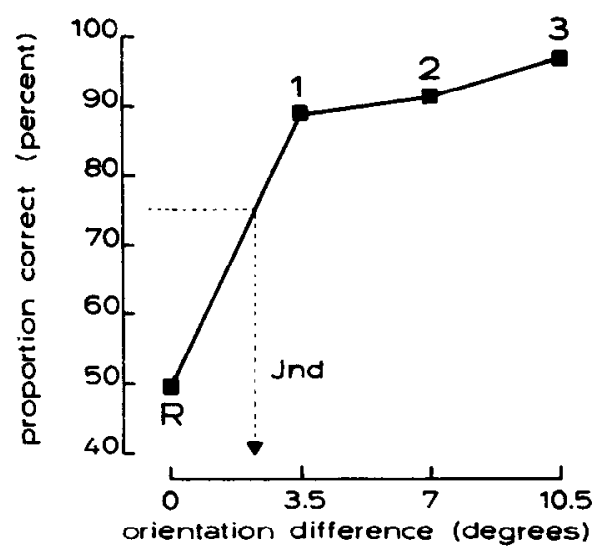

proportion of correct responses is plotted as a function of orientation, is called the neurometric curve, which is a measure of the discriminative capacity of the neuron for these pairs of orientations.

The curve shown in Figure 5 is only half of the neurometric curve corresponding to the choice of the optimal orientation as reference. One can also use the cell's firing to distinguish the reference from orientations larger than the reference (on the right of the optimum in Fig. $5 A$ ). Because, on average, the reference yields larger responses than these other orientations, the same decision criterion can be used. The complete curve for the optimal orientation as reference is given in Figure 6 (top curve). The curve is not symmetric due to the difference in steepness of the tuning and the difference in variability on the 2 sides of the optimum.

There is no particular reason to take the optimal orientation 


\section{LOE 29}

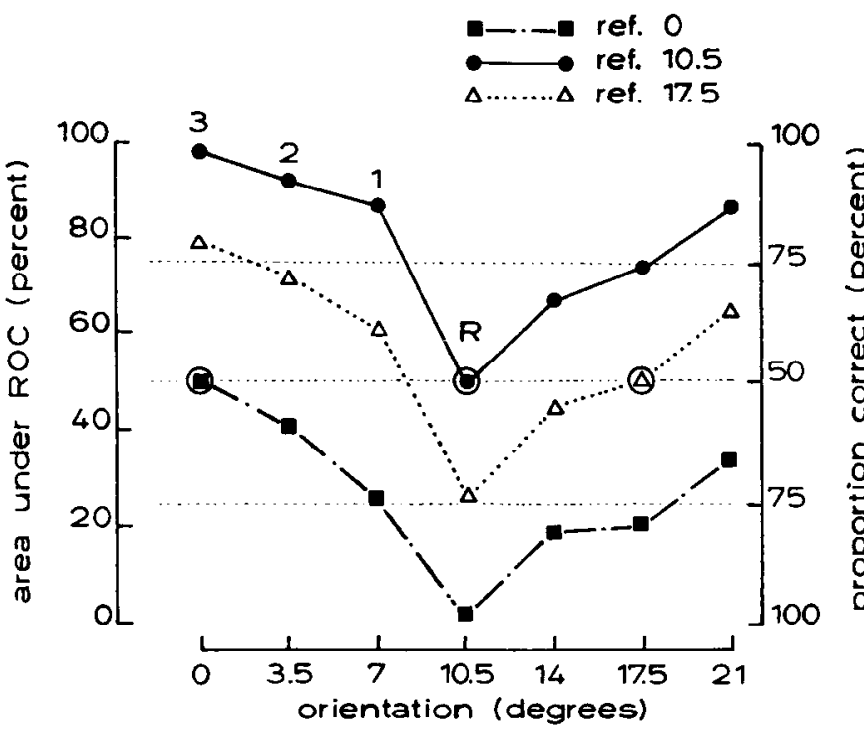

Figure 6. Neurometric curves of cell Loe 29 (same cell as shown in Fig. 5) considering 3 different orientations as reference: the optimal orientation $\left(10.5^{\circ}\right)$ as in Figure 5 (solid circles), orientation $0^{\circ}$ (solid squares), and orientation $17.5^{\circ}$ (open triangles). The left-hand ordinate indicates the area under the ROC curve for the decision rule: responses larger than the criterion represent hits for the reference orientation. The right-hand ordinate indicates the proportion of correct responses of an ideal observer using this rule for areas under the ROC larger than $50 \%$ and the reverse rule for areas under ROC smaller than 50\%. Inverting the rule amounts to inverting the ROC diagram. Both are plotted as a function of $S_{1}$ orientation. On each of the 3 neurometric curves, the reference is indicated by an open circle. Notice that the left-hand part of the upper curve is the same as the neurometric curve in Figure $5 F$. This is indicated by the labels $R, 1,2$, and 3 .

as reference. In principle, any orientation tested can be taken as reference. The neurometric curves obtained for different references are shown in Figure 6. Using orientation $0^{\circ}$ as reference but keeping the same decision rule as that used for the optimal orientation as reference will yield many more false alarms than hits for any criterion. Indeed, all other orientations evoke larger responses, on average, than orientation $0^{\circ}$ (see Fig. $5 A$ ). Hence, the ROC curves for reference $0^{\circ}$ will all lie in the lower right half of the diagram, and the area under the curves will be smaller than $50 \%$ for all orientations when compared to $0^{\circ}$ orientation as reference as indicated by the ordinate on the left-hand side of Figure 6. Reversing the decision rule and considering as hits the presentations for which the number of spikes evoked by the reference is smaller than the criterion will reverse the ROC diagram, and the ROC curves for reference $0^{\circ}$ will come to lie in the upper left quadrant. Thus, the percent of correct responses obtained by integrating the area under the curves will exceed $50 \%$. The ordinate on the right-hand side of Figure 6 captures this change in the decision rule. The upper half, ranging from 100 to $50 \%$, corresponds to the original decision rule, and the lower half, ranging from 50 to $100 \%$, corresponds to the reverse decision rule.

Until now, we have only considered as reference those orientations producing maximum or minimum responses. As a consequence, the neurometric curves lie in one or the other half of Figure 6, indicating that a single decision rule could be used for discriminating the reference from all other orientations. If an intermediate orientation of $17.5^{\circ}$ is chosen as reference, the
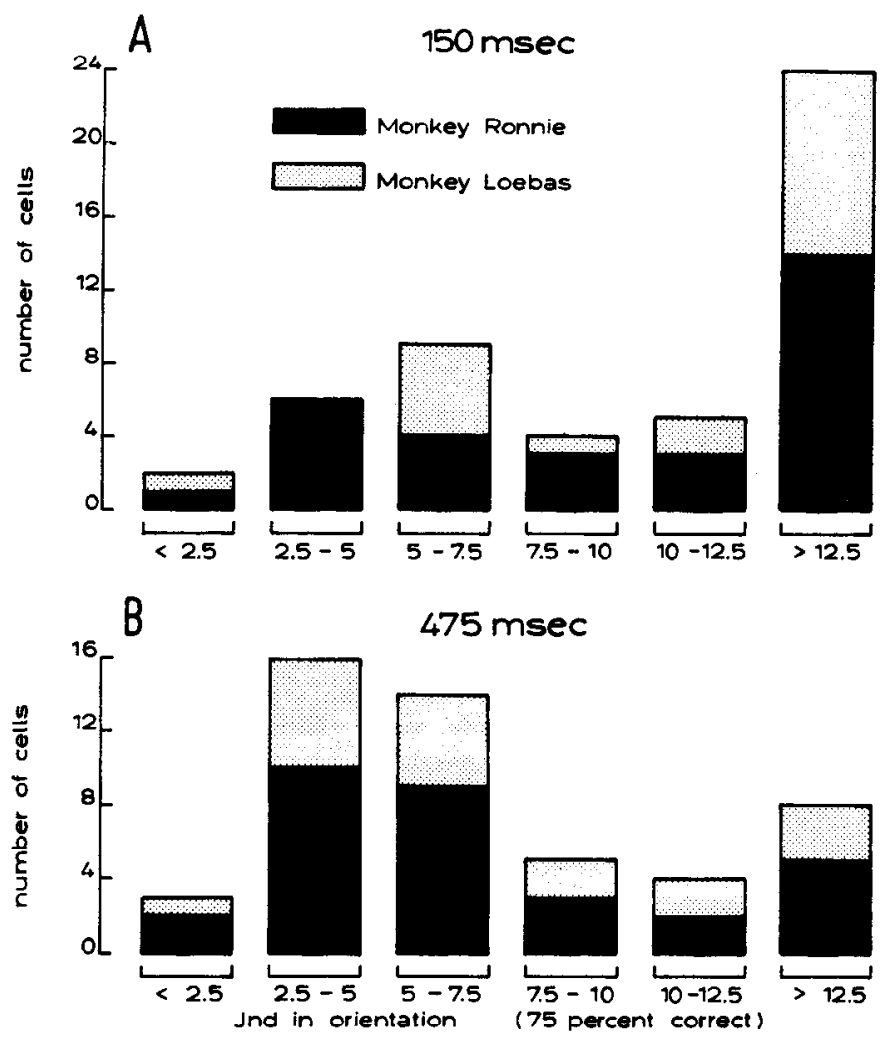

Figure 7. Distributions of neural JNDs obtained by analyzing data of variable-orientation task with fine steps and counting spikes in a short interval, $150 \mathrm{msec}(A)$, and a long interval, $475 \mathrm{msec}(B)$. For the short interval, the median is larger than $12.5^{\circ}$; for the long interval, the median is close to $6^{\circ}$. This improvement with interval duration is due to the increase in the number of spikes counted in the interval.

neurometric curve will cross from one half of Figure 6 to the other, a general feature for all references evoking intermediate responses. For the sake of clarity, the neurometric curve of only 1 intermediate reference is shown in Figure 6. If, on average, the response to the reference is larger than the response to the other orientations, the original rule should be used. In the opposite case, the reverse rule should be used. In all instances, the neurometric curve gives the probability of correct decision using these rules, either of which allows the reference orientation to be discriminated from the other orientations.

We define the neural JND as the orientation closest to the reference orientation that can be discriminated with a probability of $75 \%$ correct. It is clear from Figure 6 that this JND will depend on the reference chosen: taking $0^{\circ}$ as reference yields a JND of $6.9^{\circ}$; taking $17.5^{\circ}$ as reference yields a JND of $15.2^{\circ}$, and considering $10.5^{\circ}$ as reference yields 2.3 or $7.1^{\circ}$ as JND, depending on whether one moves counterclockwise or clockwise from the reference. For each cell, we considered in turn all orientations tested as reference, calculated all corresponding JNDs, and selected the smallest value as the neural JND of the cell. For cell Loe 29 (Fig. 6), the JND was 2.3 , obtained by the comparison of responses to orientation $7^{\circ}$ and $10.5^{\circ}$, which corresponds to the steepest part of the tuning curve with the lowest variability.

Figure $7 A$ gives the distribution of the neural JNDs of the 50 cells tested with the variable-orientation task with small steps. More than half of the cells had JNDs exceeding $10^{\circ}$. Only 2 neurons had JNDs between $2.5^{\circ}$ and $2^{\circ}$, which is close to the 
behavioral JNDs of the best monkey and humans. One of the reasons these neural JNDs are so large is the short interval on which they had to be calculated in order to take into account the short saccadic latency of monkeys. One could expect that using a longer interval would yield a larger number of spikes and hence smaller JNDs. Figure $7 B$ shows the neural JNDs obtained for the same 50 cells with a longer interval of 475 msec, starting $25 \mathrm{msec}$ and ending $500 \mathrm{msec}$ after $\mathrm{S}_{1}$ onset. This is the interval used previously by Bradlcy ct al. (1987). For this interval, $40 \%$ of the same cells have JNDs smaller than $5^{\circ}$. Comparison between the 2 parts of Figure 7 shows that the neural JND improves with increasing intervals. It also shows that the monkey does not use all the information present at the level of V1, probably because it requires him to sample neural activity for a longer time, which would increase reaction times.

\section{ROC analysis of the difference in response to successively presented stimuli signaling orientation difference}

The previous ROC analysis of the responses to $S_{1}$ in the variableorientation task with fine steps supposed that the responses to identical orientations were independent of the order of presentation. In order to test this assumption, we carried out the following analysis. For the cells tested in the variable-orientation task with coarse steps, we averaged the responses of all correct trials for conditions in which successively presented stimuli were identical. We averaged only correct trials, because on these trials, the monkey maintained fixation until the end of the stimulus presentation. For each cell, we compared for the first and second stimulus presentation the number of spikes occurring in a 300msec interval following stimulus onset, but allowing for a latency of $50 \mathrm{msec}$. The difference in spite counts was tested for significance with the nonparametric Wilcoxon test for matched pairs (2-tailed $p<0.05$ ). For monkey Loebas, only 42 cells were available for analysis as a result of a software problem, while 78 cells recorded in Ronnie were analyzed. In monkey Ronnie, $30 \%$ of the cells had significantly different responses to identical successive presentations, compared to $15 \%$ in monkey Loebas. Thus, though for the majority of cells, the assumption that identical stimuli presented in succession evoke identical responses holds, this is not true for about $25 \%$ of the $\mathrm{Vl}$ cells. In monkey Ronnie, about $90 \%$ of the cells with significantly different responses to successive identical stimuli gave smaller responses to the second stimulus compared to the first, as illustrated in the left-hand pair of histograms of Figure $8 A$ for cell Ron 26.

The responses of the variable-orientation task with fine steps were sampled over a $10-15 \mathrm{~min}$ period. Slow changes in responsiveness over this period of time will be included in the response variability and will therefore influence the neural JNDs. For the measurement of behavioral JNDs, only successively presented stimuli following each other within $650 \mathrm{msec}$ are used, and only variability over this short timespan may influence the behavioral JNDs. Hence, a fairer comparison between the neural and behavioral JNDs could be made if the neural JND was also measured using successively presented stimuli. This was exactly the purpose of the fixed-orientation task.

The ROC method chosen to analyze the fixed-orientation task data should meet 2 requirements. First, it should minimize the contribution of long-term fluctuations (fluctuations over the duration of the sampling period). Indeed, this was the very aim of the fixed-orientation task. Second, because a number of neurons (about $1 / 4$ of our V1 sample) responded unequally to successive, physically identical stimuli, the analysis should not assume equality of responses to $S_{2}$ and $S_{1}$. Three ROC analyses could, in principle, be performed on the fixed-orientation task data, but only 1 meets the 2 requirements. The first strategy would be to analyze the distribution of $S_{2}$ responses in a manner similar to that which analyzed $S_{1}$ responses. This would correspond to an absolute identification strategy in the decision process. In temporal, 2-alternative, forced-choice experiments, subjects can follow either of 2 strategies: in the absolute-identification strategy, they only take into consideration the sccond stimulus and base their decision on the representation of this stimulus; in the paired-comparison strategy, they actually compare the second stimulus with the first one on each trial (Vogels and Orban, 1986). The first type of ROC analysis would meet the second requirement but not the first. Indeed, long-term fluctuations would not be minimized. The second possibility would be to compare the distribution of $S_{2}$ responses to the distribution of $S_{1}$ responses. This analysis corresponds to neither of the 2 strategies a subject can use in 2-alternative, forced-choice situations. Furthermore, it meets neither of the 2 requirements indicated above. In particular, it will yield neurometric curves that are difficult to interpret if the responses to successive identical stimuli are different, as illustrated by the cell in Figure $8 \mathrm{~A}$. In fact, we calculated the neurometric curve for this cell using the ROC analysis of the $S_{2}$ and $S_{1}$ distributions. The percent correct for 0 orientation difference was $33 \%$ rather than the $50 \%$ required for correct estimation of the neural JND. Finally, the third analysis strategy is to calculate, on a trial-to-trial basis, the difference between $S_{2}$ and $S_{1}$ responses and analyze the distribution of these differences. Calculation of these differences increases the variability by a factor $\sqrt{2}$ or less. However, this analysis meets both requirements outlined above and, in addition, corresponds to the paired-comparison decision strategy, which is optimal under our behavioral testing methods. Therefore, we chose to perform an ROC analysis on the response-difference distributions.

Figure 8 illustrates the ROC analysis of the response differences. On average, this analysis was performed on data from 40 trials. As shown by the histograms of Figure $8 A$, the response to the second stimulus increased as the orientation difference increased from 0 to $7.5^{\circ}$. As a consequence, the response difference $\left(S_{2}-S_{1}\right)$ also increased. This was true for all cells, whether or not the response differences for zero orientation difference was positive or negative, as was the case for cell Ron 26 . Figure $8 B$ shows the distributions of the response differences for orientation differences $2.5^{\circ}$ and 0 . For 9 different criteria, the proportions of hits and false alarms are computed. A hit or false alarm is defined as the response difference $S_{2}-S_{1}$ being larger than the criterion for the nonzero orientation difference and the zero orientation difference, respectively. These proportions are illustrated for a medium criterion $\left(C_{4}\right)$ in Figure $8 B$. For this criterion, the proportion of hits was $84 \%$, and the proportion of false alarms was $30 \%$. Plotting the proportion of hits as a function of the proportion of false alarms yields the ROC curve for orientation difference $2.5^{\circ}$ (Fig. $8 C$ ). The ROC curve for zero orientation difference is the positive diagonal. The ROC curves are plotted in Figure $8 D$ for the 3 orientation differences tested $\left(2.5^{\circ}, 5^{\circ}\right.$, and $\left.7.5^{\circ}\right)$. Integrating the area under the $4 \mathrm{ROC}$ curves corresponding to the 4 orientation differences yields the neurometric curve from which a JND can be derived by taking the orientation difference corresponding to the $75 \%$-correct level. This JND was 2.3 for cell Ron 26.

We performed this ROC analysis on 33 fixed-orientation tests 
A

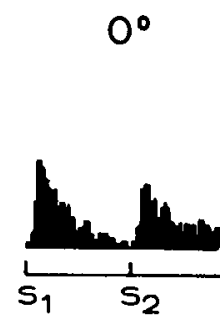

$2.5^{\circ}$

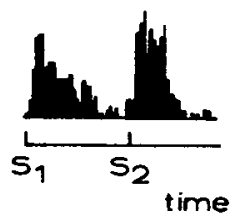

$5^{\circ}$

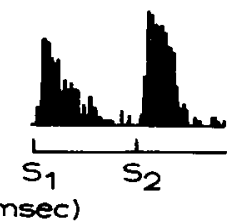

$7.5^{\circ}$

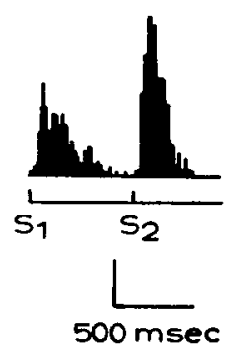

B
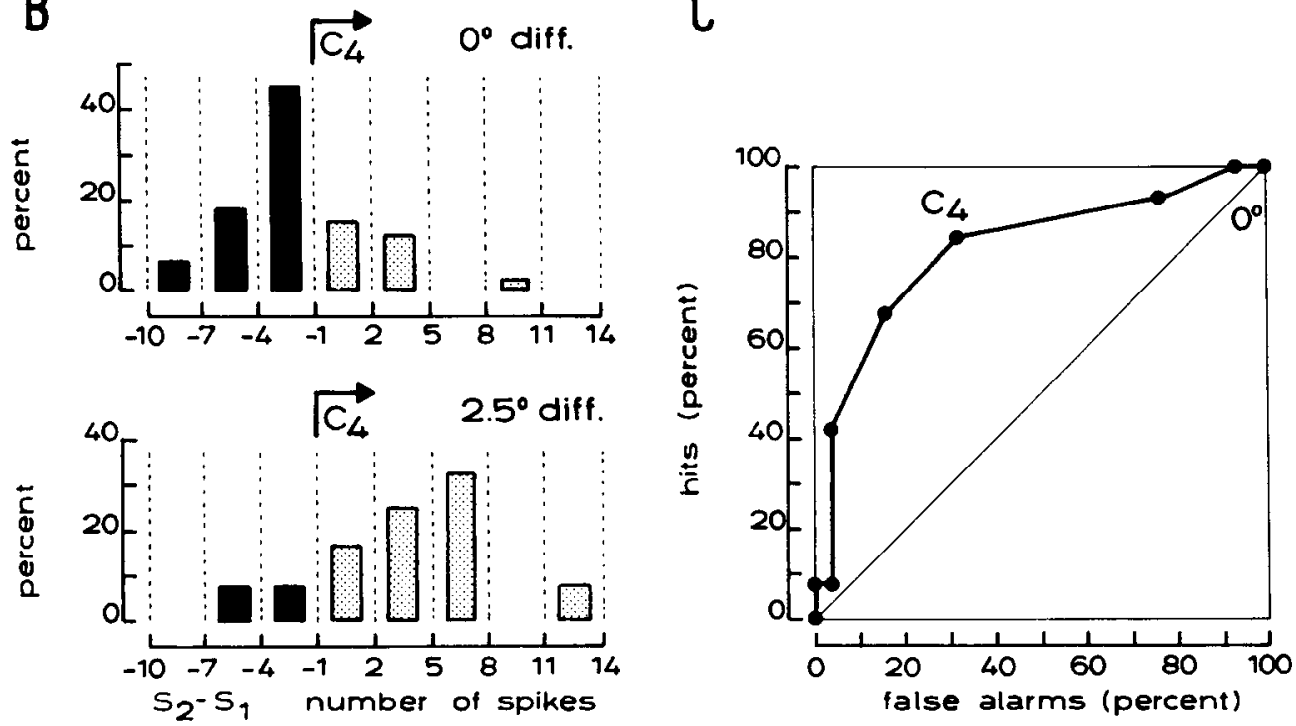

D

E

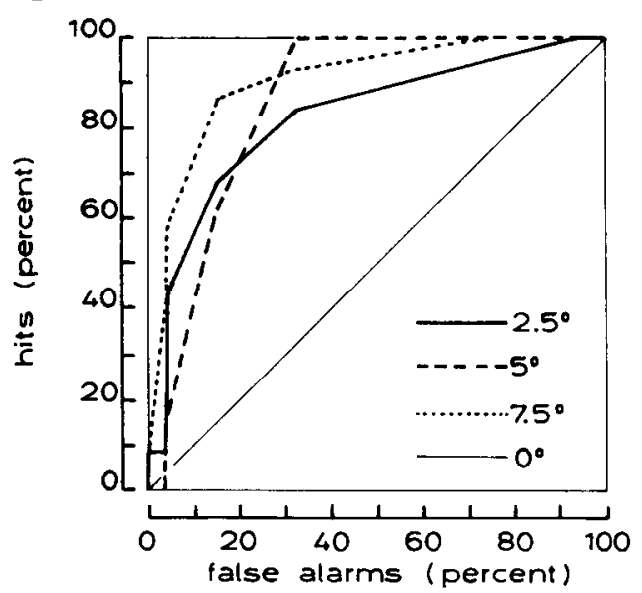

C

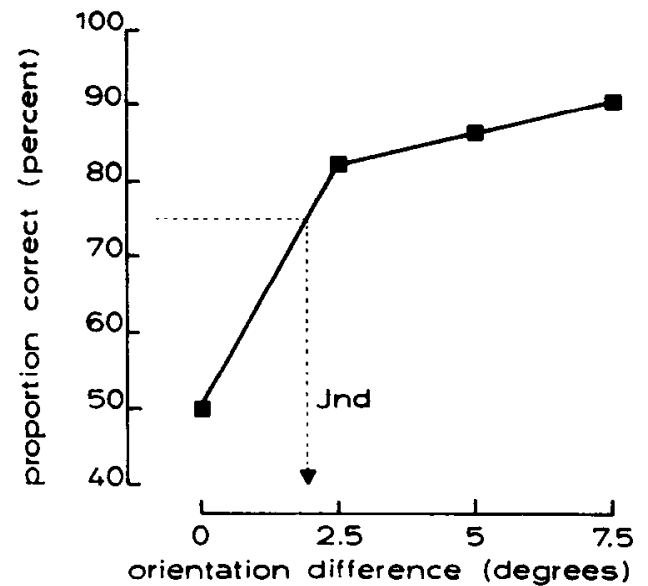

Figure 8. ROC analysis of response difference $S_{2}-S_{1}$ as function of orientation difference. $A$, Peristimulustime histograms (20-msec binwidth) representing the average firing of cell Ron 26 as a function of time for 4 orientation differences: $0^{\circ}, 2.5^{\circ}, 5^{\circ}$, and $7.5^{\circ}$. These data were obtained with the fixedorientation task at orientation $29.75^{\circ}$, in the initial part of the rising slope of the tuning curve, which is shown in Figure $10 \mathrm{~A}$ (optimum was $50.75^{\circ}$ ). Calibration bars: 38 spikes/sec vertically, $500 \mathrm{msec}$ horizontally. $B$, Distributions of the difference in response to $S_{2}$ and $\mathrm{S}_{1}$ plotted for $0^{\circ}$ and $2.5^{\circ}$ orientation difference compared to a medium level criterion $\left(C_{4}\right)$. The responses were calculated for an interval of $150 \mathrm{msec}$ starting $25 \mathrm{msec}$ after stimulus onset. The 9 criteria are indicated by vertical broken lines. The proportion of hits and false alarms for the rule that differences larger than the criterion indicate an orientation difference are marked by shaded columns. The remaining distributions are indicated by solid columns. $C$, The ROC curve for an orientation difference of $2.5^{\circ}$ derived from the distributions shown in $B$. Each of the 9 criteria contributes a point to the ROC curve. Zero orientation difference yields the positive diagonal. $D$, ROC curves obtained for $0^{\circ}, 2.5^{\circ}, 5^{\circ}$, and $7.5^{\circ}$ orientation differences. $E$, Neurometric curve plotting the proportion of correct decisions as a function of the orientation difference between $S_{1}$ and $S_{2}$. The neural JND derived from the curve is indicated. carried out on 21 cells recorded in monkey Ronnie. In those cells tested at more than 1 orientation, we selected the neurometric curve with the lowest JND as characterizing the cell. The distribution of the JNDs of these 21 cells is shown in Figure 9. More than $50 \%$ of the cells had JNDs larger than $7.5^{\circ}$. Only 3 cells had a JND between $2.5^{\circ}$ and $2^{\circ}$. These cells can signal differences in orientation of the same order as those discriminated by the best monkey.
The fixed-orientation task assesses the discriminative capacity of the cell at only 1 point on the orientation tuning curve. As was the case for the discriminative capacity assessed with the variable-orientation task using fine steps, the discriminative capacity of the cell changed with orientation. This is illustrated in Figure 10. Cell Ron 26 was tested at 5 different orientations along its tuning curve, as shown in Figure $10 \mathrm{~A}$. The neurometric curves obtained at these orientations are illustrated in Figure 


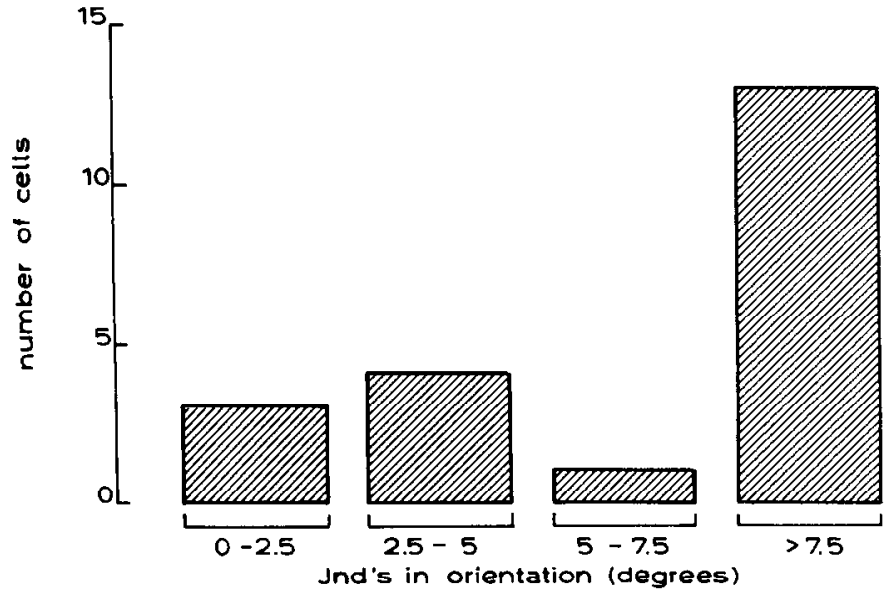

Figure 9. Distribution of neural JNDs obtained with fixed-orientation task. The JNDs were derived from neurometric curves obtained with short intervals (150 msec starting $25 \mathrm{msec}$ after stimulus onset). The median of the distribution is larger than $7.5^{\circ}$.

$10 B$. The steepest neurometric curves were obtained from orientations lying on a steep portion of the tuning curve, as was the case for orientation 1 (shown in Fig. 8) and orientation 3, both of which yielded JNDs below $2.5^{\circ}$. The shallowest neurometric curve was obtained when the orientations in the fixed test straddled the optimum orientation, as in the case of orientation 4. Other orientations gave intermediate results. These observations were fairly general, insofar as the smallest JNDs were obtained when the orientations tested lay on steep parts of the tuning curve.

\section{Comparison of the 2 ROC analyses}

Five cells were tested with both the variable-orientation task using small steps and the fixed-orientation task. The neural JNDs derived from these 2 types of tests for the 5 cells are listed in Table 3. There is an excellent agreement between the results of both analyses. For 1 cell (Ron 6), the variable-orientation test yielded a much smaller JND $\left(<2.5^{\circ}\right)$ than did the fixedorientation test $\left(>7.5^{\circ}\right)$. However, when the JND extracted from the variable-orientation test was determined at the same orientation as the one at which the fixed-orientation test was performed, a similar JND $\left(>7.5^{\circ}\right)$ was obtained. This points to a shortcoming of the fixed-orientation test mentioned above. The agreement between the results of both kinds of tests for the other 4 cells suggests that, generally, the test orientation of the fixed test was close to the steepest point of the tuning, which indeed we had attempted to do during the recording. Two cells, Ron 26 (Fig. 8) and Ron 32, had significantly different responses to $S_{1}$ and $S_{2}$ when both stimuli were identical. Yet, the JNDs derived from both kinds of tests were in good agreement. This suggests that the differences in response between successive identical stimuli, which occurred only in $1 / 4$ of the cells, did not appreciably influence the results obtained in the variable-orientation test.

The variability measured in the variable-orientation task with small steps includes both a short-tcrm component measured over an interval less than $1 \mathrm{sec}$ and a long-term component due to slow fluctuations in responsiveness during the 10-15-min test session. The variability measured in the fixed-orientation task consists only of the short-term component. If both tests yield similar JNDs, this indicates that the long-term component of the variability under our conditions is relatively negligible. Analysis of the responses in the fixed-orientation task showed that this was indeed the case.

In order to separate short- and long-term components of the response variability, the following analysis was performed on the 33 fixed-orientation tests of the 21 cells recorded in monkey Ronnie. If $V(1), V(2)$, and $V(1-2)$ represent the variance of the response to $S_{1}$, to $S_{2}$, and of the difference between the 2 responses, respectively, the following holds:

$$
V(1-2)=V(1)+V(2)-2 \operatorname{COV}(1,2),
$$

where $\operatorname{COV}(1,2)$ stands for the covariance of the responses to successive identical-stimulus presentations. This covariance factor can be taken as an index of long-term variability, because this variability will necessarily result in correlation between the responses to successive stimuli. Because $V(1), V(2)$, and $V(1-$ 2) could be determined from our data, the covariance factor could be calculated using the formula given above. The variances were determined for the number of spikes occurring in the 150-msec interval starting $25 \mathrm{msec}$ after stimulus onset. Most cells showed long-term fluctuations in their responses. Indeed, in about $3 / 4$ of the 33 tests, $V(1-2)$ was smaller than the sum of $V(1)$ and $V(2)$, indicating that the covariance differed from zero. However, these long-term fluctuations were only a minor component of the total variance. Indeed, the median proportion of the variance arising from long-term fluctuations was only $15 \%$ (first quartile, $-6 \%$; third quartile, $23 \%$ ).

\section{Tuning characteristics determining the neural JND}

The neural JND was smallest when the orientations tested lay on the steep parts of the tuning curve, whether estimated in the variable- or the fixed-orientation task. Therefore, it is not surprising that our data show no correlation between neural JNDs and orientation bandwidth. In order to investigate which tuning characteristics the neural JNDs depend upon, we divided the 50 cells tested with the variable-orientation task using fine steps into 2 subgroups, with JNDs below and above $7.5^{\circ}$. We then calculated which of the tuning characteristics or combinations thereof most clearly separated cells with small and large JNDs. The result of this analysis is shown in Figure 11. This figure depicts the cumulative frequencies of the tuning characteristics for the 2 populations of cells. While bandwidth was similar for cells with small and large JNDs, response strength was larger, and normalized variance was smaller in cells with small JNDs compared to cells with large JNDs. When these differences were tested with a $\chi^{2}$ test on the frequency distributions, none were significant, though the $\chi^{2}$ was larger for the normalized variance $\left(\chi^{2}=3.3, d f=2, p>0.05\right)$ and response strength $\left(\chi^{2}=4.2, d f\right.$ $=2, p>0.05)$ than for bandwidth $\left(\chi^{2}=1.13, d f=2, p>0.05\right)$. The response strength divided by the bandwidth, a factor proportional to the slope of the tuning curve, distinguished the 2 cell populations better than any single characteristic. The difference between the 2 populations was significant at the $5 \%$ level $\left(\chi^{2}=6.67, d f=2\right)$. The best discrimination was obtained by considering all 3 characteristics and dividing response strength by bandwidth and normalized variance. For this factor, the difference between cells with small and large JNDs was significant at the $0.5 \%$ level $\left(\chi^{2}=12.1, d f=2\right)$. Similar tendencies were observed when this analysis was performed on the cells recorded in Loebas and Ronnie separately, as well as in the sample of cells tested in the fixed-orientation task. The number 


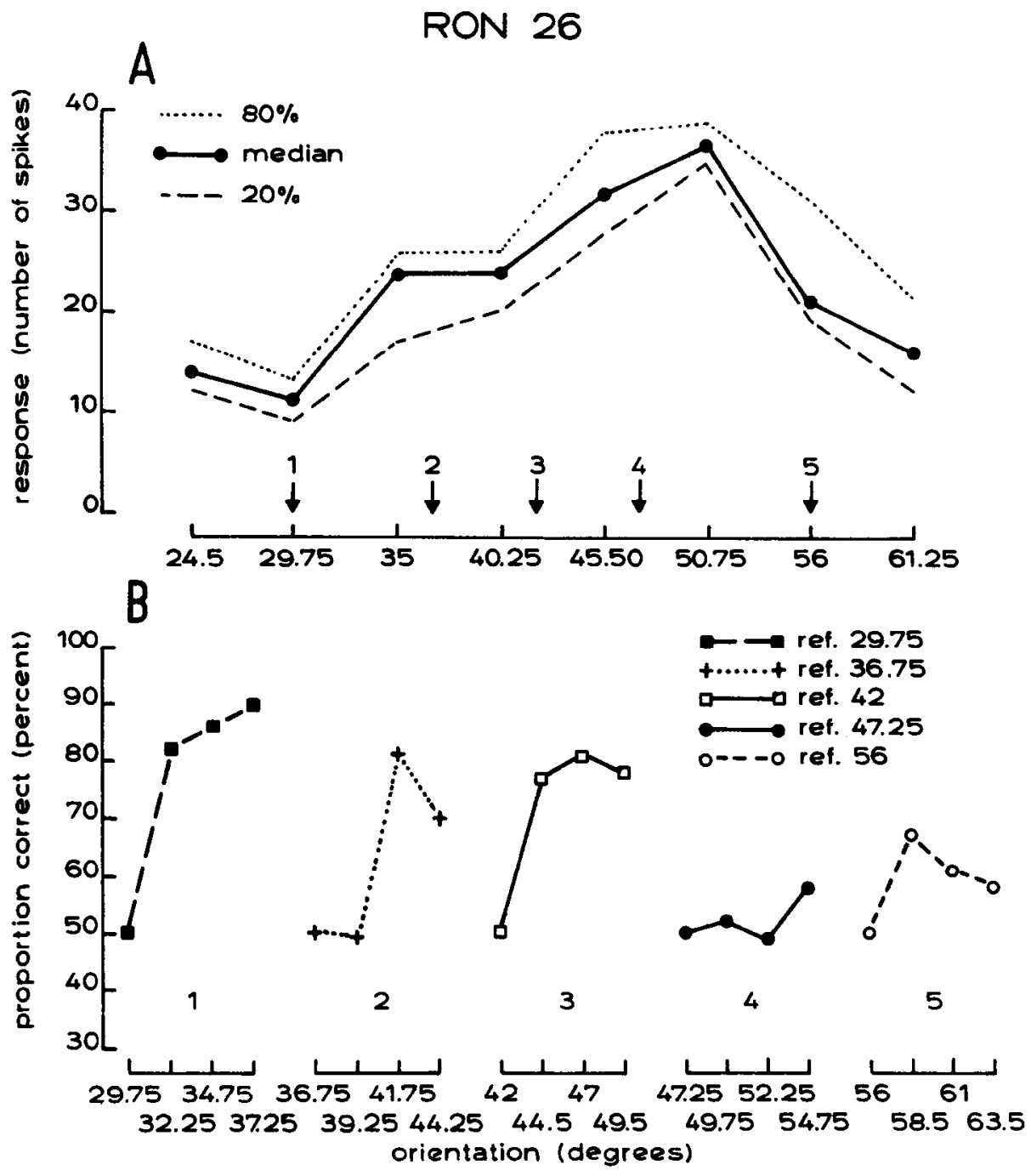

Figure 10. Comparison of neurometric curvcs obtained with fixed-orientation task at different reference orientations. The same cell is shown as in Figure 8. $A$, Orientation tuning curve: the median number of spikes counted in a 500 -msec interval after $S_{1}$ onset is plotted as a function of $S_{1}$ orientation. The 20th and 80th percentiles are indicated, as well as the 5 orientations at which the fixed-orientation test was performed. $B$, Neurometric curves obtained at 5 different orientations (labeled $1-5$ ) for a short analysis interval $(150 \mathrm{msec})$. In order to facilitate the comparison with the tuning curve, the neurometric curves are plotted as a function of the orientation of $S_{2}$. The neurometric curve obtained at the orientation labeled 1 is the same as that of Figure 8.

or cells in each of these samples was too small, however, to test for statistical significance with the $\chi^{2}$ test.

\section{Discussion}

We investigated the ability of single V1 cells to signal small differences in orientation by small but reliable differences in firing rate under conditions similar to those under which behavioral JNDs are measured. We used an ROC procedure (Bradley et al., 1987; Newsome et al., 1989) in 50 cells to analyze the response changes with stimulus orientation. In 21 cells, we used a novel ROC analysis of differences in response to successive stimuli as a function of orientation difference. The first analysis corresponds to an absolute-identification decision strategy, though applied to $S_{1}$ rather than to $S_{2}$ because of the larger number of trials available for $S_{1}$. The second analysis corresponds to a paired-comparison strategy, which is optimal under our behavioral conditions of testing. The 2 kinds of analyses are in good agreement. This is demonstrated by the similarity of the JNDs obtained when the 2 tests are applied to the same cell. On first glance, this seems to be contradicted by the distributions of JNDs obtained for the 2 samples. The fixed-orientation task seems to yield a somewhat higher proportion of cells with a JND similar in magnitude to the behavioral JND than does the variable-orientation task. For the latter task, only
2 of 50 cells had JNDs below $2.5^{\circ}$, while 3 of 21 cells tested in the fixed task had JNDs below 2.5 . However, this difference can easily be accounted for by the stronger selection of the fixedorientation sample. Indeed, those cells clearly had steeper slopes in their orientation tuning and less variability than did the sample tested in the variable-orientation task.

Therefore, our experiments clearly show that a minor fraction of V1 cells can signal differences in orientation of about the same magnitude as those that our best monkey and human can discriminate. This result was obtained by recording from monkeys while they were performing an orientation-discrimination task. The stimulus, a stationary grating, was identical in either situation, and the analysis interval was adapted to the short

\begin{tabular}{|c|c|c|}
\hline Cell & JND, fixed & JND, variable \\
\hline Ron 6 & $>7.5$ & $<2.5(>7.5)$ \\
\hline Ron 26 & $<2.5$ & 3.3 \\
\hline Ron 32 & 3.8 & 3 \\
\hline Ron 80 & $>7.5$ & $>7.5$ \\
\hline Ron 83 & $>7.5$ & $>7.5$ \\
\hline
\end{tabular}



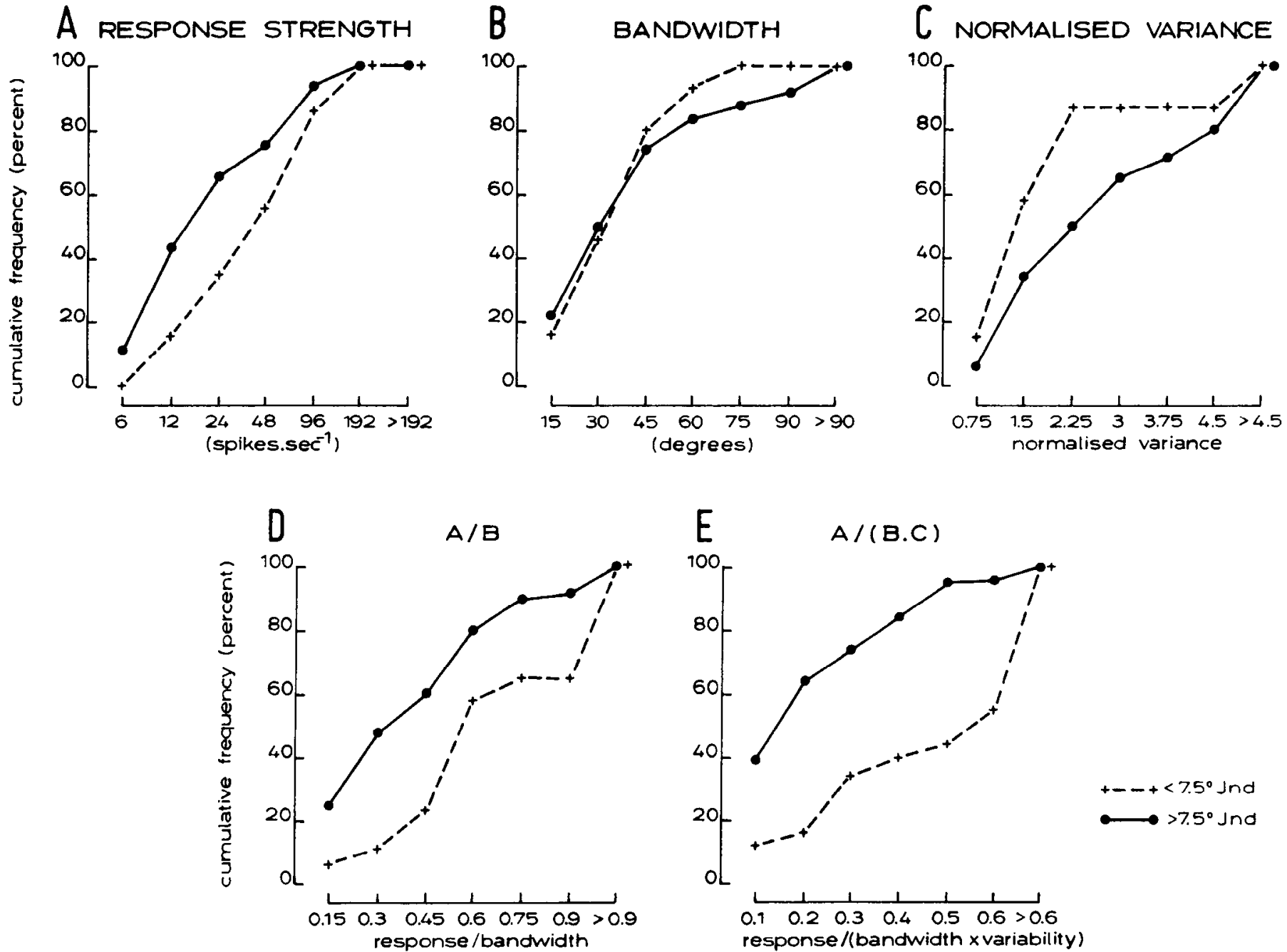

Figure 11. Cumulative frequencies (integrals of frequency distributions) of orientation-tuning characteristics and their combinations for neurons with small JNDs $\left(<7.5^{\circ}\right.$, crosses) and with large JNDs $\left(>7.5^{\circ}\right.$, solid circles). The JNDs were derived from the variable-orientation task with fine steps. $A$, Response strength. $B$, Bandwidth. $C$, Normalized variance. $D$, Response strength divided by bandwidth, indicating the slope of the tuning curve. $E$, Response strength divided by bandwidth and normalized variance. The distance between the cumulative frequency curves of the 2 populations is a measure of how much these populations differed for a given characteristic.

reaction times of monkeys. Despite these efforts to record under conditions matching as closely as possible those under which behavioral JNDs are measured, a direct comparison of neural and behavioral JNDs remains difficult for several reasons. First, in our experiments, the 2 types of JNDs are measured at different instances in time. A second factor is that, behaviorally, the 2 monkeys had different performances. Since these experiments were completed, we have trained a third monkey, which had JNDs of $2.1^{\circ}$, averaged over the 3 reference orientations. This suggests that the JNDs of Ronnie reflect more typically the thresholds of monkeys performing well in the discrimination task, but that a certain proportion of monkeys has difficulties achieving this fine level of discrimination. In the cat, the same seems to be true because, out of a large sample of 26 cats, about $1 / 4$ of the cats failed to achieve the fine discriminations the remaining 3/4 achieved (P. De Weerd, E. Vandenbussche, and G. A. Orban, unpublished observations). As a consequence, JNDs in this population of cats ranged from $2^{\circ}$ to $14^{\circ}$. Also, in humans, JNDs can vary considerably when large numbers of subjects are tested. Vogels et al. (1984) observed that JNDs for oblique references ranged from $1.2^{\circ}$ to $12.8^{\circ}$ and JNDs for principal references ranged from $0.6^{\circ}$ to $3.2^{\circ}$ in a population of 18 normal young adults. Finally, there was a difference between the stimuli in the 2 experimental situations in that, during behavioral testing, the grating phase was randomly shifted, while the phase was fixed in the physiological experiments. Once the monkey was well trained, behavioral JNDs were the same, whether or not phase was randomized; hence, this difference does not affect the comparison of behavioral and neural JNDs. Furthermore, in the 3 cells in which we randomized the stimulus phase, the variability was the same with or without randomization.

The degree to which we can compare neural and behavioral JNDs is also influenced by our choice to use standard stimuli and not a stimulus optimized for each cell under study. In particular, one could argue that we should have optimized the phase of the grating while stabilizing the eye position. We felt that we should not attempt to optimize the phase of the fixed grating, just as we did not attempt to adapt dimensions, depth, or even color of the grating to the characteristics of the cell under study. Indeed, this would mean that the question at hand concerns the smallest difference in orientation that visual cortical cells can signal under conditions optimal for each cell. We think it is 
more meaningful to try to answer the question of how small a difference in orientation cortical cells can signal in the standard conditions under which behavioral measures are taken. The first reason is that it might not be technically feasible to optimize all stimulus parameters for the cell under study. Some of the stimulus requirements of cells might even be unknown. Second, cells that respond poorly in these standard conditions will also contribute little to the behavior, because both measures are taken under the same conditions. Finally, systematic, and in many cases, random variations of stimulus parameters have little effect on the behavioral JNDs. In case of grating phase, random variation does not affect behavioral JNDs; neither do random changes in contrast or position of the grating (Burbeck and Regan, 1983; Paradiso et al., 1989). This means either that the monkey must base its perception on those cells that, at each trial, are optimally stimulated, or that in the visual pathway, a representation of orientation is gradually emerging that is invariant for parameters such as grating phase, position, and contrast. The first alternative is extremely unlikely because it is difficult to see how the monkey would select, on each trial, the cells that are optimally stimulated. The use of the same standard stimulus facilitates the comparison between performance of cells in different cortical areas in which cells have different stimulus requirements. This should allow us to explore the second alternative and to follow the emergence of an invariant representation of stimulus orientations along the visual pathway. That this is indeed possible is demonstrated by our recent observation that, under the same standard conditions under which we recorded from V1 cells, inferotemporal cells respond vigorously and in an orientation-selective way to gratings (Orban and Vogels, 1990).

Finally, the comparison between neural and behavioral JNDs is influenced by the variability in eye position in our experiments. While the relatively large dimension of the grating compared to the RF dimensions in V1 ensured that the RF was always covered by the stimulus, the variability in eye position will increase the response variability of phase-sensitive cells. Hence, their neural JND will be overestimated. This, in our opinion, does not affect the comparison of the behavioral JND with the neural JND of the phase-sensitive cell if we make this comparison to decide whether the activity of a single neuron would suffice to explain the behavior. As indicated below, however, there are many reasons to believe that the behavior reflects the activity of an ensemble of neurons, rather than that of a single cell. If we would use our data to estimate how well an ensemble of neurons with the characteristics measured in our experiments can represent orientation, we would, because of the overestimation of variability, underestimate the trial-by-trial contribution of phase-sensitive cells to the ensemble coding.

It is difficult to compare our results to those of Bradley et al. (1987). Not only did these authors record from anesthetized and paralyzed cats, but we have little information concerning the tuning characteristics of their 22 cells. They mention that the average bandwidth of these cells was $29^{\circ}$, not dissimilar to the average of our samples, but they provide no average values for response strength or variance, which also determine the performance of cells. These authors used a long interval, $500 \mathrm{msec}$, to calculate the spike distributions on which the ROC analysis was performed. Cats can make discriminations as quickly as $350 \mathrm{msec}$ after stimulus onset (De Weerd et al., 1990), and a shorter interval would therefore have been more realistic. One of the reasons for reanalyzing our data with a 475 -m $\mathrm{mec}$ interval starting $25 \mathrm{msec}$ after the stimulus onset was to compare them to those of Bradley et al. (1987). This comparison shows that the 2 distributions of neural JNDs are similar, with an average close to $6^{\circ}$ in either case. Furthermore, our results confirm Bradley et al.'s (1987) finding that the JND depends on position along the tuning curve and is optimal at those points where the tuning curve is steepest.

Despite our efforts to make the physiological recording conditions as similar as possible to those under which behavioral thresholds are measured and to define the interval during which the single-cell activity is measured as precisely as possible, 2 uncertainties that influence the estimation of neural JNDs remain. As previous authors have done (Bradley et al., 1987; Newsome et al., 1989; Parker and Hawken, 1985; Swindale and Cynader, 1986), we simply counted the number of spikes occurring during an interval after stimulus onset. This assumes that the temporal characteristics of the spike train contain no additional information. Some authors have argued that there are stimulus-locked temporal modulations of single-cell responses that could provide additional information (Strehler and Lestienne, 1986; Richmond et al., 1987). It is possible that these temporal modulations result from the way the system, a network, produces single-cell responses, and that they do not constitute additional information for the system. Furthermore, the short interval used in our analysis limits the number of temporal codes that could be used by the monkey visual system. Nonetheless, if the system uses a temporal code that is more efficient than the average firing code, the neural JNDs of single cells may well be lower than those reported here. The other assumption of our ROC analysis is that the spike trains are 'observed' by an ideal detector that is noise free and uses the optimal-decision rule. Furthermore, the second ROC analysis assumes that there is a perfect noise-free memory store available to save the spike count evoked by the first stimulus presentation for comparison with the second stimulus. In humans, at least, it has been shown that decision and memory factors contribute to the global variance that determines the orientation threshold (Vogels and Orban, 1986). It is therefore likely that the neural JNDs are in fact overestimations of the single cell's contribution to the perceptual decision. The second ROC analysis also implies that the monkey can somehow compute the difference in response to $S_{1}$ and $S_{2}$ on a single trial (paired-comparison strategy). This does not mean that it has to memorize the distribution of response differences, but that it has to compute this difference on each trial and compare it to a criterion. This assumption has little incidence on the estimation of neural JNDs.

Even if the most-performing cells in V1 have neural JNDs of the order of behavioral JNDs, one cannot escape the conclusion that there is a large discrepancy between individual V 1 cells and the observable behavior. First, the behavioral performance of the 2 monkeys was very different, yet the properties of cells in V1 were relatively similar. Bandwidth and response strength were comparable for the cells recorded in the 2 animals, but normalized variance and latency were larger in monkey Loebas than in monkey Ronnie. Because the reaction time of Loebas was also longer than that of Ronnie, the difference in normalized variance of the $V 1$ cells alone would have to explain a difference in behavioral JND of a factor of 3 . Second, our results show that more information could be extracted from the firing of single V 1 cells by waiting longer before deciding, but the monkey clearly does not follow this strategy. Third, though some neural JNDs are on the order of those measured behaviorally, none 
was smaller than $1^{\circ}$, a value measured regularly for human observers tested at principal orientations and observed occasionally in monkey Ronnie. Fourth, in humans, the JNDs are smaller at principal orientations than at an oblique orientation, and 1 month of training can reduce much of this difference (Vogels and Orban, 1985). The difference between human and monkey subjects may be a species difference or may be due to the overtraining of the monkeys. The training effects are difficult to explain by the properties of $\mathrm{V} 1$ cells because the evidence for adult plasticity in V1 is very scant. Finally, behavioral JNDs are unaffected by random changes in other stimulus dimensions such as contrast, spatial frequency, and position in humans (Burbeck and Regan, 1983; Paradiso et al., 1989) and monkeys (R. Vogels and G. A. Orban, unpublished observations), while $\mathrm{V} 1$ cells' activity clearly depends on these parameters. It is therefore very likely that orientation is signaled not by the activity of an individual V1 cell, but by the interaction between an ensemble of V 1 cells (Vogels and Orban, 1989a). Yet, the question remains of whether perception is based directly on the activity of an ensemble of V1 cells or on the activity of an ensemble of cells in higher-order areas receiving from V1. Lesion studies from our laboratory (Vandenbussche et al., 1989; Orban et al., 1990) suggest that a subprimate species such as the cat may have adopted the former strategy, and that there is no further cortical processing of orientation beyond arcas 17 and 18 . In primates, it seems that the other alternative applies, and that there is further cortical processing of the stimulus orientation beyond V1. Given the large position invariance of orientation discrimination in humans (Paradiso et al., 1989), an area such as inferotemporal cortex, which has large RFs extending well into the ipsilateral hemifield, could be involved in primates. Indeed, lesions of inferotemporal cortex impair orientation discrimination in the monkey (Dean, 1978) and, very recently, we have recorded orientation-selective responses of IT neurons in monkey Ronnie (Orban and Vogels, 1990). This indeed suggests that simple visual parameter discriminations such as orientation discrimination are a useful tool for studying the entire primate visual system.

\section{References}

Bradley A, Skottun BC, Ohzawa I, Sclar G, Freeman R (1987) Visual orientation and spatial frequency discrimination: a comparison of single neurons and behavior. J Neurophysiol 57:755-772.

Burbeck CA, Regan D (1983) Independence of orientation and size in spatial discriminations. J Opt Soc Am 73:1691-1694.

Cohn TE, Green DG, Tanner WP Jr (1975) Receiver operating characteristic analysis: application to the study of quantum fluctuation effects in optic nerve of Rana pipiens. J Gen Physiol 66:583-616.

Dean P (1978) Visual cortex ablations and thresholds for successively presented stimuli in rhesus monkeys: 1. Orientation. Exp Brain Res $32: 445-458$

De Valois RL, Yund EW, Hepler N (1982) The orientation and direction selectivity of cells in macaque visual cortex. Vision Res 22: 531-544.

De Weerd P, Vandenbussche PE, Orban GA (1990) Bar orientation discrimination in the cat. Visual Neurosci 4:257-268.

Ellaway PH (1978) Cumulative sum technique and its application to the analysis of peristimulus time histograms. EEG Clin Neurophysiol 45:302-304.

Green DM, Swets JA (1966) Signal detection theory and psychophysics. New York: Wiley.

Judge SJ, Richmond BJ, Chu FC (1980) Implantation of magnetic search coils for measurement of eye positions: an improved method. Vision Res 20:535-538.
Newsome WT, Britten KH, Movshon JA (1989) Neuronal correlates of a perceptual decision. Nature 341:52-54.

Orban GA, Vogels R (1990) Orientation selectivity of inferotemporal neurons: a study in the discriminating monkey. Invest Opthalmol Vis Sci 31:90.

Orban GA, Vandenbussche E, Vogels R (1984) Human orientation discrimination tested with long stimuli. Vision Res 24:121-128.

Orban GA, Vandenbussche E, Sprague JM, De Weerd P (1988) Stimulus contrast and visual cortical lesions. Exp Brain Res 72:191-194.

Orban GA, Vandenbussche E, Sprague JM, De Weerd P (1990) Orientation discrimination in the cat: a distributed function. Proc Natl Acad Sci USA 87:1134-1138.

Paradiso MA, Carney T, Freeman RD (1989) Cortical processing of hyperacuity tasks. Vision Res 29:247-254.

Parker A, Hawken M (1985) Capabilities of monkey cortical cells in spatial resolution tasks. J Opt Soc Am A2:1101-1114.

Poggio GF, Doty RW, Talbot WH (1977) Foveal striate cortex of behaving monkey: single-neuron responses to square-wave gratings during fixation of gaze. J Neurophysiol 40:1369-1391.

Richmond BJ, Optican LM, Podell M, Spitzer H (1987) Temporal encoding of two-dimensional patterns by single units in primate inferior temporal cortex. J Neurophysiol 57:132-146.

Robinson DA (1972) Eye movements by collicular stimulation in the alert monkey. Vision Res 12:1795-1808.

Rocha-Miranda CE, Bender DB, Gross CG, Mishkin M (1975) Visual activation of neurons in inferotemporal cortex depends on striate cortex and forebrain commissures. J Neurophysiol 38:475-491.

Schiller PH, Finlay BL, Volman SF (1976) Quantitative studies of single-cell properties in monkey striate cortex. II. Orientation specificity and ocular dominance. J Neurophysiol 39:1320-1333.

Skottun BC, Bradley A, Sclar G, Ohzawa I, Freeman RD (1987) The effects of contrast on visual orientation land spatial frequency discrimination: a comparison of single cells and behavior. J Neurophysiol 57:773-786.

Strehler BL, Lestienne R (1986) Evidence on precise time-code symbols and memory of patterns in monkey cortical neuronal spike trains. Proc Natl Acad Sci USA 83:9812-9816.

Suzuki H, Azuma M (1976) A glass insulated 'Elgiloy' microelectrode for recording unit activity in chronic monkey experiments. EEG Clin Neurophysiol 41:93-95.

Swindale NV, Cynader MS (1986) Vernier acuity of neurons in cat visual cortex. Nature 319:591-593.

Tolhurst DJ, Movshon JA, Dean AF (1983) The statistical reliability of signals in single neurons in cat and monkey visual cortex. Vision Res 23:775-785.

Vandenbussche E, Orban GA (1983) Meridional variations in the line orientation discrimination of the cat. Behav Brain Res 9:237-255.

Vandenbussche E, Vogels R, Orban GA (1986) Human orientation discrimination: changes with eccentricity in normal and amblyopic vision. Invest Ophthalmol Vis Sci 27:237-245.

Vandenbussche E, Sprague JM, De Weerd P, Orban GA (1989) Effect of higher cortical ablations on cat orientation discrimination. Soc Neurosci Abstr 15:1255.

Vogels R, Orban GA (1985) The effect of practice on the oblique effect in line orientation judgements. Vision Res 25:1679-1687.

Vogels R, Orban GA (1986) Decision processes in visual discrimination of line orientation. J Exp Psychol [Hum Percept] 12:115-132.

Vogels R, Orban GA (1989a) Linking striate cortical cells and orientation discrimination: a study in the behaving monkey. Perception 18:551.

Vogels R, Orban GA (1989b) Orientation discrimination thresholds of single striate cells in the discriminating monkey. Soc Neurosci Abstr 15:324.

Vogels R, Orban GA, Vandenbussche E (1984) Meridional variations in orientation discrimination in normal and amblyopic vision. Invest Ophthalmol Vis Sci 25:720-728.

Vogels R, Spileers W, Orban GA (1989) The response variability of striate cortical neurons in the behaving monkey. Exp Brain Res 77: 432-436.

Wetherill GB, Levitt H (1965) Sequential estimation of points on a psychometric function. Br J Math Stat Psychol 18:1-10. 